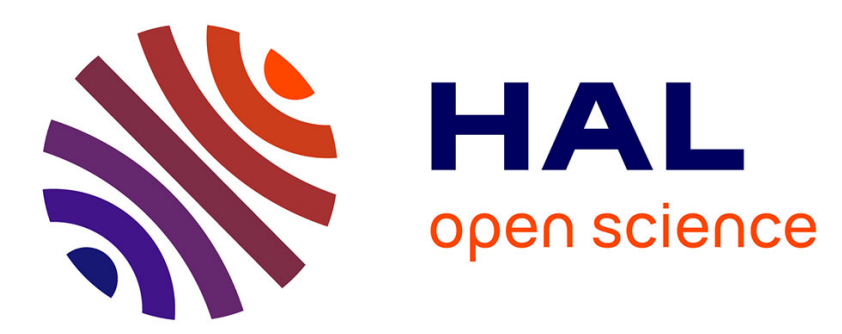

\title{
Low-rank Factorizations in Data Sparse Hierarchical Algorithms for Preconditioning Symmetric Positive Definite Matrices
}

\author{
Emmanuel Agullo, Eric Darve, Luc Giraud, Yuval Harness
}

\section{To cite this version:}

Emmanuel Agullo, Eric Darve, Luc Giraud, Yuval Harness. Low-rank Factorizations in Data Sparse Hierarchical Algorithms for Preconditioning Symmetric Positive Definite Matrices. [Research Report] RR-9200, Inria Bordeaux Sud-Ouest. 2018. hal-01856399v2

\section{HAL Id: hal-01856399 \\ https://hal.inria.fr/hal-01856399v2}

Submitted on 27 Aug 2018

HAL is a multi-disciplinary open access archive for the deposit and dissemination of scientific research documents, whether they are published or not. The documents may come from teaching and research institutions in France or abroad, or from public or private research centers.
L'archive ouverte pluridisciplinaire HAL, est destinée au dépôt et à la diffusion de documents scientifiques de niveau recherche, publiés ou non, émanant des établissements d'enseignement et de recherche français ou étrangers, des laboratoires publics ou privés. 
Low-rank Factorizations in Data Sparse Hierarchical Algorithms for Preconditioning Symmetric Positive Definite Matrices

Emmanuel Agullo, Eric Darve, Luc Giraud, Yuval Harness

RESEARCH REPORT

$\mathrm{N}^{\circ} 9200$

August 2018

Project-Team HiePACS 



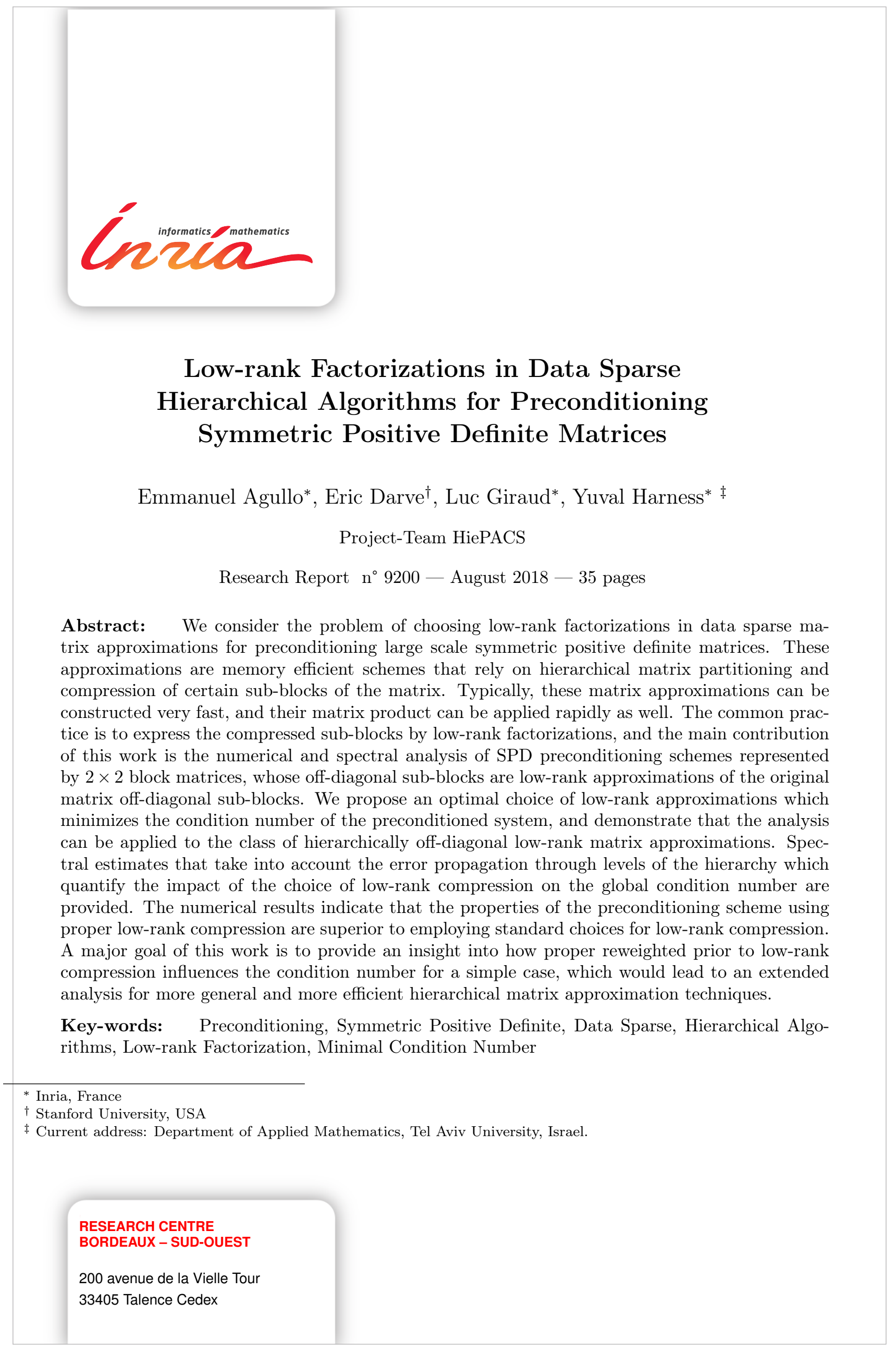




\section{Factorisation de rang faible pour des algorithmes hiérarchique pour préconditionner des matrices symétriques définies positives}

Résumé : Nous étudions un préconditionnement "data sparse" pour matrices symétriques définies positives provenant de problèmes aux limites elliptiques du second ordre. Pour des problèmes de grandes tailles, des conditions aux limites quasi-singulières ou des géomètries complexes, les matrices de discrétisation associées sont très mal conditionnées. Le recours à des méthodes multi-niveaux sont souvent une nécessité pour obtenir une solution efficace. Nous proposons un nouveau préconditionneur hiérarchique qui, dans le cas deux niveaux, mimise le conditionnement du système préconditionné. Dans le cas multi-niveau le preconditionneur tente de conserver cette propriété qui n'est plus prouvée; en revanche nous établissons que les valeurs propres extrémales sont clusterisées dans un intervalle autour de 1. Finalement, à travers des expérimentations numériques, nous illustrons l'efficacité du nouveau schéma proposé qui surpasse les techniques plus classiques basées sur une SVD régulière pour approximer les blocs hors-diagonaux ou une SVD filtrée.

Mots-clés : Solveurs directs rapides, préconditionnement, matrices hiérarchiques, matrices creuses, matrice de rang faible, compression hiérarchique 


\section{Contents}

\begin{tabular}{llr}
\hline 1 & Introduction & 3 \\
\hline
\end{tabular}

2 The Optimal One-level Preconditioning Scheme 5

2.1 Explicit Formula of the Optimal One-level Scheme . . . . . . . . . . . . . . . . . 6

2.2 Minimal Condition Number and Spectral Analysis . . . . . . . . . . . . . . . . 6

2.3 Proof of Theorem 1. . . . . . . . . . . . . . . . . . . 8

3 The Multilevel Weighted HODLR Preconditioning Scheme 11

3.1 Symmetric HODLR Matrix Structure . . . . . . . . . . . . . . . . . . . . 12

3.2 Recursive Formula of the Multilevel Preconditioning Scheme. . . . . . . . . . . . 13

3.3 Utilization and Construction of the Preconditioning Scheme . . . . . . . . . . . . 14

3.4 Spectral Estimates and Error Propagation . . . . . . . . . . . . . . . . . . . 15

3.5 Proof of Theorem $2 \ldots \ldots \ldots \ldots \ldots \ldots \ldots \ldots \ldots$

\begin{tabular}{lll}
4 & Numerical Study & 22 \\
\hline
\end{tabular}

4.1 Poisson Problem with Robin Boundary Conditions . . . . . . . . . . . . . . . . . 23

4.2 Subdomain Preconditioning Simulation . . . . . . . . . . . . . . . . . . . . 23

4.3 Sparse Matrix Simulations . . . . . . . . . . . . . . . . . . . . . . . . . 25

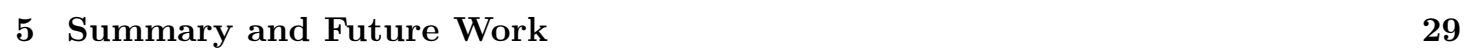

\section{Introduction}

In this paper we consider preconditioning for iterative solution of large scale linear systems

$$
A x=b,
$$

where $A \in \mathbb{R}^{n \times n}$ is a symmetric positive definite (SPD) matrix. Such systems arise in a wide range of engineering applications, as means to model and understand physical phenomena. Typical example is the result of a finite element discretization of underlying differential equations of a boundary value problem. In many practical applications the matrix $A$ becomes ill-conditioned and, thus, challenging for iterative techniques. In that case the use of preconditioned iterative methods, such as the preconditioned conjugate gradient (PCG) [22, 31] technique, becomes an imperative. The choice of a suitable preconditioning scheme can, often, drastically improve the convergence behavior of the iterative method and, generally, plays a vital role in the success of solving the system.

A preconditioning scheme for linear systems is, essentially, composed of linear operations or matrices that approximate $A^{-1}$ eq. 1.1 , but with considerable less computational effort than explicitly inverting $A$. Transforming the system eq. (1.1) with such a scheme is called the preconditioned system. The major concern when setting up a preconditioning scheme is to ensure that the preconditioned system has a bounded condition number, and that the number of iterations to convergence in an iterative method remains small while maintaining low associated complexity and reduced memory cost. The literature on preconditioning techniques is vast, and many robust and efficient methods have been introduced in the last 50 years. These include, among others, incomplete factorization schemes such as ILU and Incomplete Cholesky, sparse matrix approximations, polynomial techniques, domain decomposition methods, as well as multigrid and algebraic multilevel iterations schemes. For a recent comprehensive review on this topic see [33]. 
The main contribution of this work is the numerical and spectral analysis of SPD preconditioning schemes represented by $2 \times 2$ block matrices, whose off-diagonal sub-blocks are low-rank approximations of the original matrix off-diagonal sub-blocks. We re-examine the way low-rank factorizations are obtained, by considering reweighting of the sub-blocks prior to the low-rank compression. Reweighting can be done in many ways, e.g., diagonal scaling, and the fundamental question that we attempt to answer is: which reweighting is optimal with respect to the condition number of the preconditioned system?

The mathematical theory for $2 \times 2$ matrices is derived in section 2 . We present an optimal 1-level preconditioning scheme using proper reweighting prior to low-rank compression, which minimizes the spectral condition number of the preconditioned system. Thus, a preconditioning scheme employing such low-rank factorizations is expected to attain the same condition number with less computational resources and associated complexity, compared to employing other standard techniques for the low-rank factorizations. Spectral analysis shows that the scheme maps both small and large eigenvalues of the original system exactly to 1 . This feature is of great importance to Krylov subspace methods, since it is equivalent to the minimization of the effective degree of the minimal polynomial of $A$ that defines the maximal dimension of the search space.

In section 3 we propose an application of the 1-level theory for hierarchically off-diagonal low-rank (HODLR) matrix structure, as means to demonstrate the applicability of the 1-level theory to the hierarchical multilevel case. We also provide spectral estimates that take into account the error propagation through levels of the hierarchy. This leads to quantification of the impact of the reweighting on the global condition number of the preconditioned system. In essence, weighted HODLR locally minimizes the condition number at each level of the hierarchy by approximately filtering the smallest and largest eigenvalues. Since this approach is employed hierarchically, it effectively creates a strong effect of global spectrum clustering.

The HODLR structure is a member of a wide class of hierarchical data sparse approximations. These approximations rely on the fact that the matrix can be sub-divided into a hierarchy of smaller block matrices, and certain sub-blocks can be efficiently approximated as low-rank matrices by low-rank factorizations. The low-rank compressions of sufficient sub-blocks leads to a dramatic reduction of the complexity and computational cost. The best known example for such schemes is the class of hierarchical matrix ( $\mathcal{H}$-matrix) approximations [17, 19, 20, 5] which has gained growing attention in recent years.

To the contrary of the more general strong hierarchical matrix structure which allows further decomposition of the off-diagonal blocks into low-rank and full-rank blocks, HODLR is a weak hierarchical matrix structure, which relies on a single low-rank compression for the off-diagonal blocks. Closely related to HODLR is the hierarchically semi-separable (HSS) [8, 35] structure , which is, in fact, a HODLR matrix format possessing a nested off-diagonal low-rank structure.

Essentially, weak hierarchical methods, i.e., HODLR and HSS, are not considered competitive with the more general strong hierarchical matrix methods, when the underlying problem is of very large scale. However, the proposed study provides a novel theoretical basis for optimality conditions of hierarchical preconditioning schemes. Thus, the presented analysis can serve as starting point for a more general theory on optimal $\mathcal{H}$-matrix preconditioning which is deferred to future work.

The weighted HODLR scheme is similar in nature to the methods proposed in 36, 37. which propose practical HSS schemes that rely on similar ideas of reweighting prior to compression, but without the complete numerical and spectral analysis of this study. The costs to apply these multilevel preconditioners are about $\mathcal{O}(n)$, where $n$ is the matrix size.

The experimental part of this work, whose goal is to demonstrate the effectiveness of properly chosen reweighting for the low-rank approximations, is given in section 4 The section contains a 
detailed comparative study of HODLR preconditioning using different methods for the low-rank compressions. As alternatives to the proper reweighting strategy, we consider the conventional low-rank approximation in the 2-norm and the low-rank approximation with constraints [6]. The latter employs low-rank approximations that also preserve constraints, forcing sub-blocks of the preconditioning scheme to be identical to the corresponding sub-blocks of the input matrix on predetermined subspaces. Employing the method for preconditioning SPD matrices of discretized elliptic PDEs has been demonstrated in [7, and a similar approach for non-symmetric sparse matrices has been recently suggested in 38 .

The numerical results indicate, that employing proper reweighting prior to low-rank compression, leads to a HODLR preconditioning scheme that requires far less computational resources for the same quality of convergence performance compared to using other low-rank compression techniques. The experiments also show, that the HODLR preconditioning scheme with proper reweighting retains the SPD property of the system when other standard techniques fail, and remains efficient and robust even if low accuracy compression is employed with ranks of $\mathcal{O}(1)$ for the low-rank approximations of the sub-blocks. Summary and plans for future work follow in section 5 .

\section{The Optimal One-level Preconditioning Scheme}

In this section we introduce the optimal 1-level scheme for the preconditioning of SPD matrices. We consider an input $n \times n$ SPD matrix $A$ with a $2 \times 2$ block structure and a corresponding 1-level approximation $K$,

$$
A=\left[\begin{array}{cc}
A_{1} & M \\
M^{T} & A_{2}
\end{array}\right], \quad K=\left[\begin{array}{cc}
A_{1} & U_{1} S V_{2}^{T} \\
V_{2} S U_{1}^{T} & A_{2}
\end{array}\right], \quad A_{i} \in \mathbb{R}^{n_{i} \times n_{i}},
$$

where $n=n_{1}+n_{2}$, and the off-diagonal blocks of $K$ are low-rank factorizations satisfying

$$
U_{1} \in \mathbb{R}^{n_{1} \times r}, \quad S \in \mathbb{R}^{r \times r}, \quad V_{2} \in \mathbb{R}^{n_{2} \times r},
$$

with a, typically, small rank $r$. The matrix $U_{1}$ is the interpolation operator, the matrix $V_{2}$ is the anterpolation operator, and the matrix $S$ whose rank is $r$ is known as the interaction operator. In some cases $S$ is omitted, i.e., equivalently represented by an $r \times r$ identity matrix.

We present an explicit formula for a 1-level approximation, $K$ eq. (2.1), which minimizes the spectral condition number

$$
\text { cond }\left(R^{-T} A R^{-1}\right)=\left\|R^{-T} A R^{-1}\right\|_{2} \cdot\left\|R A^{-1} R^{T}\right\|_{2},
$$

of the preconditioned system,

$$
R^{-T} A R^{-1} x=R^{-T} y
$$

for any given rank $r=0,1, \ldots, \min \left\{n_{1}, n_{2}\right\}$, where $\|\cdot\|_{2}$ is the 2-norm, and $R$ denotes any square root (not necessarily principal) of $K$ in the sense that

$$
K=R^{T} R \in \mathbb{R}^{n \times n} .
$$

The key idea is to reweight the off-diagonal blocks prior the low-rank factorization. Proper choice of reweighting leads to a minimum spectral condition number of the preconditioned system as well as clustering of the spectrum of the preconditioned system around 1 .

We begin in section 2.1 by introducing the method for obtaining the minimum condition number low-rank approximation. In section 2.2 we provide the theorem on the minimum condition number property, including a detailed description of the spectral properties of the preconditioned system. A rigorous and detailed proof of the theorem is given in section 2.3 


\subsection{Explicit Formula of the Optimal One-level Scheme}

The construction of the minimum condition number 1-level preconditioner $K$ eq. 2.1 subject to

$$
\operatorname{rk}\left(U_{1} S V_{2}^{T}\right) \leq r
$$

is based on the following two-step method ensuring that the preconditioned matrix $R^{-T} A R^{-1}$ also inherits the SPD property of $A$ :

1. Apply a two-sided block Jacobi transformation,

$$
\left[\begin{array}{cc}
R_{1}^{-T} & 0 \\
0 & R_{2}^{-T}
\end{array}\right] \cdot A \cdot\left[\begin{array}{cc}
R_{1}^{-1} & 0 \\
0 & R_{2}^{-1}
\end{array}\right]=\left[\begin{array}{cc}
I_{1} & R_{1}^{-T} M R_{2}^{-1} \\
R_{2}^{-T} M^{T} R_{1}^{-1} & I_{2}
\end{array}\right]
$$

where $I_{i}$ denotes the $n_{i} \times n_{i}$ identity matrix, and $R_{i} \in \mathbb{R}^{n_{i} \times n_{i}}$ denotes a square root of $A_{i}$ i.e., $R_{i}^{T} R_{i}=A_{i}$.

2. Extract the off-diagonal triple products eq. (2.2) by setting,

$$
U_{1}=R_{1}^{T} \mathcal{U}_{r}, \quad S=\Sigma_{r}=\operatorname{diag}\left(\sigma_{1}, \ldots, \sigma_{r}\right), \quad V_{2}=R_{2}^{T} \mathcal{V}_{r}
$$

where $\mathcal{U}_{r}$ and $\mathcal{V}_{r}$ are composed of the first $r$ left and right, respectively, singular vectors of the singular value decomposition (SVD),

$$
R_{1}^{-T} M R_{2}^{-1}=\mathcal{U} \Sigma \mathcal{V}^{T}, \quad \Sigma=\operatorname{diag}\left(\sigma_{1}, \ldots, \sigma_{\min \left\{n_{1}, n_{2}\right\}}\right) .
$$

The theory presented in this study implies, that in practice for a given rank bound $r \geq 0$, any low-rank factorization, $U_{1} S V_{2}^{T}$, satisfying

$$
\left\|R_{2}^{-T} M^{T} R_{1}^{-1}-R_{2}^{-T} U_{1} S V_{2}^{T} R_{1}^{-1}\right\|_{2} \leq\left\|R_{2}^{-T} M^{T} R_{1}^{-1}-\mathcal{U}_{r} \Sigma_{r} \mathcal{V}_{r}^{T}\right\|_{2},
$$

would achieve the same minimal spectral condition number. However, the truncated SVD of the reweighted off-diagonal block, $\mathcal{U}_{r} \Sigma_{r} \mathcal{V}_{r}^{T}$, also ensures that the spectrum of the preconditioned system is optimally clustered. This observation is discussed and explained in the next subsection.

\subsection{Minimal Condition Number and Spectral Analysis}

Let us now focus on the spectral properties of the preconditioned system, $R^{-T} A R^{-1}$, where $R$ is a square root of $K$ eq. (2.1) whose off-diagonal low-rank blocks are given by eq. (2.8). First, let us consider the degenerate case $r=0$. In this case $U_{1} S V_{2}^{T}=0$ and the square root of $K$ reduces to the following block diagonal form,

$$
R(r=0)=\left[\begin{array}{cc}
R_{1} & 0 \\
0 & R_{2}
\end{array}\right]
$$

The preconditioning scheme eq. (2.4) with $r=0$ is, in fact, the two-sided block Jacobi eq. (2.7). There is a known result [12 showing that the two-sided block Jacobi preconditioner eq. 2.7] is optimal, in the sense that

$$
\operatorname{cond}\left(\left[\begin{array}{cc}
R_{1}^{-T} & 0 \\
0 & R_{2}^{-T}
\end{array}\right] A\left[\begin{array}{cc}
R_{1}^{-1} & 0 \\
0 & R_{2}^{-1}
\end{array}\right]\right) \leq \operatorname{cond}\left(\left[\begin{array}{cc}
B_{1}^{T} & 0 \\
0 & B_{2}^{T}
\end{array}\right] A\left[\begin{array}{cc}
B_{1} & 0 \\
0 & B_{2}
\end{array}\right]\right)
$$

for any non-singular $\left[\begin{array}{cc}B_{1} & 0 \\ 0 & B_{2}\end{array}\right]$ with the same dimensions and partition as $R(r=0)$ eq. 2.9 . The analysis we present, thus, naturally extends this classic result. 
The main results for the general case $r \geq 0$ are presented in theorem 1 , whose principal component is the spectral analysis of the preconditioned system. Our proof shows that the spectrum of the two-sided block Jacobi preconditioned system eq. 2.7) contains (or equals to)

$$
1+\sigma_{1}, \ldots, 1+\sigma_{\min \left\{n_{1}, n_{2}\right\}}, 1-\sigma_{\min \left\{n_{1}, n_{2}\right\}}, \ldots, 1-\sigma_{1}
$$

where $1-\sigma_{1}$ and $1+\sigma_{1}$ are the smallest and largest, respectively, eigenvalues of the preconditioned system. Thus, the two-sided block Jacobi redistributes the spectrum of the matrix symmetrically around 1. We show that the optimal 1-level preconditioning scheme does the same, but also maps the largest $r$ eigenvalues $\left(1+\sigma_{1}, \ldots, 1+\sigma_{r}\right)$ and the smallest $r$ eigenvalues $\left(1-\sigma_{r}, \ldots, 1-\sigma_{1}\right)$ of eq. (2.7) exactly to 1 . Hence, the spectral condition number eq. (2.3) as a function of $r$ is

$$
\text { cond }\left(R^{-T} A R^{-1}\right)=\frac{1+\sigma_{r+1}}{1-\sigma_{r+1}} \text {. }
$$

An illustration of the spectral clustering done by the optimal 1-level preconditioning scheme is displayed in fig. 1 .

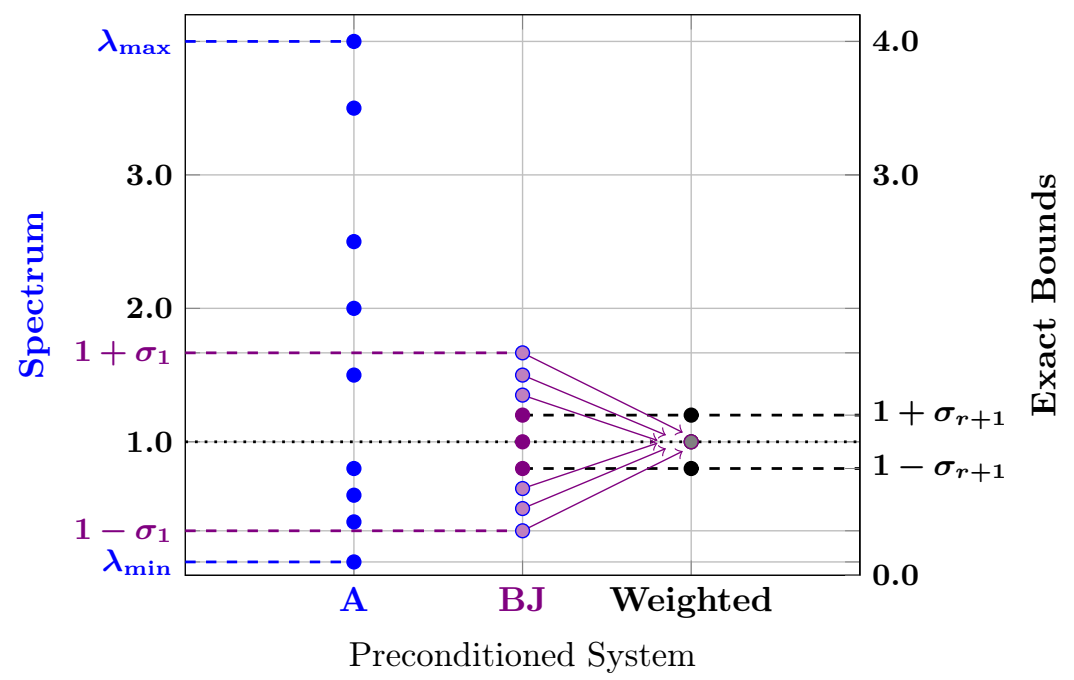

Figure 1: Spectrum Clustering of the Optimal 1-level Preconditioning Scheme. The spectrum of some SPD matrix $A$ and the transformations it goes after preconditioning by block Jacobi (BJ) and the optimal 1-level preconditioning scheme are displayed. The spectra are ordered from the left to the right starting from $A$, followed by BJ and end up with the optimal scheme.

Theorem 1. Let

$$
A=\left[\begin{array}{cc}
A_{1} & M \\
M^{T} & A_{2}
\end{array}\right], \quad K=\left[\begin{array}{cc}
A_{1} & U_{1} S V_{2}^{T} \\
V_{2} S U_{1}^{T} & A_{2}
\end{array}\right],
$$

have the same dimensions and partition where $A$ is SPD, and let $R_{i}$ denote a square root of $A_{i}$, i.e., $A_{i}=R_{i}^{T} R_{i}$.

If the off-diagonal triple product approximation $U_{1} S V_{2}^{T}$ satisfies

$$
U_{1}=R_{1}^{T} \mathcal{U}_{r}, \quad S=\Sigma_{r}, \quad V_{2}=R_{2}^{T} \mathcal{V}_{r}
$$


where $\mathcal{U}_{r}$ and $\mathcal{V}_{r}$ are composed of the first $r$ left and right, respectively, singular vectors of the SVD,

$$
R_{1}^{-T} M R_{2}^{-1}=\mathcal{U} \Sigma \mathcal{V}^{T}, \quad \Sigma=\operatorname{diag}\left(\sigma_{1}, \ldots, \sigma_{\min \left\{n_{1}, n_{2}\right\}}\right),
$$

then:

1. The matrix $K$ is $S P D$ and possesses a square root, $R$, i.e., $K=R^{T} R$.

2. For any $r<\min \left\{n_{1}, n_{2}\right\}$ and any square root $R$, the spectrum of the preconditioned system is contained in $] 0,2[$ and equal to

$$
\left\{1+\sigma_{r+1}, \ldots, 1+\sigma_{\min \left\{n_{1}, n_{2}\right\}}, 1,1-\sigma_{\min \left\{n_{1}, n_{2}\right\}}, \ldots, 1-\sigma_{r+1}\right\} .
$$

3. Any inverse square root of $K, R^{-1}$, is a minimizer of the spectral condition number eq. (2.3) in the sense that

$$
\text { cond }\left(R^{-T} A R^{-1}\right) \leq \operatorname{cond}\left(\widehat{R}^{-T} A \widehat{R}^{-1}\right), \quad \widehat{R}^{T} \widehat{R}=\widehat{K},
$$

for any SPD matrix with the same dimensions and partition as $K$ of the form,

$$
\widehat{K}=\left[\begin{array}{ll}
A_{1} & \widehat{M} \\
\widehat{M}^{T} & A_{2}
\end{array}\right],
$$

whose off-diagonal blocks satisfy $\operatorname{rk}(\widehat{M}) \leq r$.

\subsection{Proof of Theorem 1}

The proof of theorem 1 regarding the spectral properties relies on lemma 1 , while the minimum condition number property is based on the Cauchy (eigenvalue) interlacing theorem [28, p. 202]. The latter asserts that the eigenvalues of any principal submatrix of a symmetric matrix interlace those of the symmetric matrix. To be precise, if $H \in \mathbb{R}^{n \times n}$ is a partitioned symmetric matrix of the following form

$$
H=\left[\begin{array}{cc}
E & F \\
F^{T} & G
\end{array}\right]
$$

in which $E$ is a $r \times r$ principal submatrix, then for each $i=1, \ldots, r$,

$$
\lambda_{i}(H) \geq \lambda_{i}(E) \geq \lambda_{i+n-r}(H),
$$

where the eigenvalues of the symmetric matrix $H$ are assumed to be arranged in a decreasing order:

$$
\lambda_{1}(H) \geq \lambda_{2}(H) \geq \cdots \geq \lambda_{n}(H) .
$$

Lemma 1. Let $H=\left[\begin{array}{cc}\delta I_{1} & \mathcal{M} \\ \mathcal{M}^{T} & \delta I_{2}\end{array}\right] \in \mathbb{R}^{\left(n_{1}+n_{2}\right) \times\left(n_{1}+n_{2}\right)}$ where $I_{i}$ is the $n_{i} \times n_{i}$ identity matrix and $\delta \in \mathbb{R}$, and let $\sigma_{1}, \ldots, \sigma_{\min \left\{n_{1}, n_{2}\right\}}$ denote the singular values of $\mathcal{M}$.

1. If $n_{1}=n_{2}$ then

$$
\operatorname{spec}(H)=\left\{\delta-\sigma_{1}, \ldots, \delta-\sigma_{n_{1}}, \delta+\sigma_{n_{1}}, \ldots, \delta+\sigma_{1}\right\}
$$

2. If $n_{1} \neq n_{2}$ then

$$
\operatorname{spec}(H)=\left\{\delta-\sigma_{1}, \ldots, \delta-\sigma_{\min \left\{n_{1}, n_{2}\right\}}, \delta+\sigma_{\min \left\{n_{1}, n_{2}\right\}}, \ldots, \delta+\sigma_{1}\right\} \cup\{\delta\},
$$

where the multiplicity of the eigenvalue $\delta$ is at least $\left|n_{1}-n_{2}\right|$. 
Proof. of lemma 1

Let us assume without the loss of generality that $n_{1} \geq n_{2}=m$ and let

$$
\mathcal{M}=\mathcal{U} \Sigma \mathcal{V}^{T}, \quad \mathcal{U} \in \mathbb{R}^{n_{1} \times n_{2}}, \quad \mathcal{V} \in \mathbb{R}^{n_{2} \times n_{2}},
$$

denote the SVD of $\mathcal{M}$. Let

$$
\mathcal{W}=\frac{1}{\sqrt{2}}\left[\begin{array}{cc}
\widetilde{\mathcal{U}} & \mathcal{U} \\
\widetilde{\mathcal{V}} & -\mathcal{V}
\end{array}\right] \in \mathbb{R}^{n \times n}
$$

whose blocks are given by

$$
\widetilde{\mathcal{U}}=\left\{\begin{array}{rll}
\mathcal{U} & \text { if } & n_{1}=n_{2} \\
{\left[\mathcal{U} \sqrt{2} \mathcal{U}^{\perp}\right]} & \text { if } & n_{1}>n_{2}
\end{array} \quad, \quad \widetilde{\mathcal{V}}=\left\{\begin{array}{rll}
\mathcal{V} & \text { if } & n_{1}=n_{2} \\
{[\mathcal{V} 0]} & \text { if } & n_{1}>n_{2}
\end{array},\right.\right.
$$

where $\mathcal{U}^{\perp}$ is an $n_{1} \times\left(n_{1}-n_{2}\right)$ matrix with orthonormal columns, whose range is orthogonal to the range of $\mathcal{U}$,

$$
\mathcal{U}^{T} \mathcal{U}^{\perp}=0 \in \mathbb{R}^{n_{2} \times\left(n_{1}-n_{2}\right)} .
$$

Direct calculations show that $\mathcal{W}$ is an orthonormal matrix satisfying

$$
\mathcal{W}^{T}\left[\begin{array}{cc}
0 & \mathcal{M} \\
\mathcal{M}^{T} & 0
\end{array}\right] \mathcal{W}=\left[\begin{array}{cc}
\mathcal{S}_{1} & 0 \\
0 & -\mathcal{S}_{2}
\end{array}\right]
$$

where $\mathcal{S}_{i}=\operatorname{diag}\left[\sigma_{1}, \ldots, \sigma_{\min \left\{n_{1}, n_{2}\right\}}, 0, \ldots, 0\right] \in \mathbb{R}^{n_{i} \times n_{i}}$. Thus, by the orthogonality of $\mathcal{W}$ we obtain

$$
\mathcal{W}^{T}\left[\begin{array}{cc}
\delta I_{1} & \mathcal{M} \\
\mathcal{M}^{T} & \delta I_{2}
\end{array}\right] \mathcal{W}=\left[\begin{array}{cc}
\delta I_{1}+\mathcal{S}_{1} & 0 \\
0 & \delta I_{2}-\mathcal{S}_{2}
\end{array}\right]
$$

Hence, the spectrum of $H$ is given by

$$
\operatorname{spec}(H)=\left\{\delta-\sigma_{1}, \ldots, \delta-\sigma_{\min \left\{n_{1}, n_{2}\right\}}, \delta+\sigma_{\min \left\{n_{1}, n_{2}\right\}}, \ldots, \delta+\sigma_{1}\right\} \cup\{\delta\},
$$

where the multiplicity of $\delta$ is at least $n_{1}-n_{2}$. Note that in case $n_{2}>n_{1}$, we can simply interchange the principal blocks of $H$ by reordering the columns and rows of $H$, and repeat the proof.

Proof. of theorem 1

Let $\widehat{K}$ be a partitioned SPD matrix with the same dimensions and partition as $K$ eq. 2.1) whose off-diagonal blocks rank is bounded by $r$,

$$
\widehat{K}=\left[\begin{array}{cc}
A_{1} & U_{1} S V_{2}^{T} \\
V_{2} S U_{1}^{T} & A_{2}
\end{array}\right], \quad \operatorname{rk}\left(U_{1} S V_{2}^{T}\right) \leq r .
$$

If $(\lambda, \zeta) \in \mathbb{R} \times \mathbb{R}^{n}$ is an eigenpair of the preconditioned matrix

$$
\widehat{R}^{-T} A \widehat{R}^{-1}, \quad \widehat{K}=\widehat{R}^{T} \widehat{R}
$$

then by employing the change of variables, $\zeta=\widehat{R} \xi$, we obtain

$$
\widehat{R}^{-T} A \widehat{R}^{-1} \zeta=\lambda \zeta \Leftrightarrow \widehat{R}^{-T} A \xi=\lambda \widehat{R} \xi \Leftrightarrow \widehat{R}^{-1} \widehat{R}^{-T} A \xi=\lambda \xi
$$

Since $\widehat{R}^{-1} \widehat{R}^{-T}=\widehat{K}^{-1}$, we conclude that regardless to the particular choice of square root, $\widehat{R}$, the spectrum of the preconditioned system eq. 2.13 remains unchanged.

$\mathrm{RR} \mathrm{n}^{\circ} 9200$ 
Let $R_{i} \in \mathbb{R}^{n_{i} \times n_{i}}$ denote a, generally, non-symmetric square root of $A_{i}$. By direct calculations we obtain

$$
\left[\begin{array}{cc}
R_{1}^{-T} & 0 \\
0 & R_{2}^{-T}
\end{array}\right] A\left[\begin{array}{cc}
R_{1}^{-1} & 0 \\
0 & R_{2}^{-1}
\end{array}\right]=\left[\begin{array}{cc}
I_{1} & R_{1}^{-T} M R_{2}^{-1} \\
R_{2}^{-T} M^{T} R_{1}^{-1} & I_{2}
\end{array}\right],
$$

and by lemma 1 , the spectrum of eq. 2.14 is contained in (or equal to)

$$
\left\{1+\sigma_{1}, \ldots, 1+\sigma_{\min \left\{n_{1}, n_{2}\right\}}, 1,1-\sigma_{\min \left\{n_{1}, n_{2}\right\}}, \ldots, 1-\sigma_{1}\right\}
$$

where $\sigma_{i}$ are the singular values of $R_{1}^{-T} M R_{2}^{-1}$. Since $R_{i}$ are non-singular, the preconditioned matrix eq. 2.14 is SPD. Hence, we have

$$
\left.1-\sigma_{1}>0 \Rightarrow \operatorname{spec}\left(\left[\begin{array}{cc}
I_{1} & R_{1}^{-T} M R_{2}^{-1} \\
R_{2}^{-T} M^{T} R_{1}^{-1} & I_{2}
\end{array}\right]\right) \subset\right] 0,2[.
$$

Consider the specific choice of inverse square root of $\widehat{K}$,

$$
\widehat{R}^{-1}=\left[\begin{array}{cc}
R_{1}^{-1} & 0 \\
0 & R_{2}^{-1}
\end{array}\right] \widehat{\mathcal{W}} \widehat{\mathcal{D}}^{-1 / 2} \widehat{\mathcal{W}}^{T}, \quad \widehat{\mathcal{D}}=\left[\begin{array}{cc}
I_{1}+\widehat{\mathcal{S}}_{1, r} & 0 \\
0 & I_{2}-\widehat{\mathcal{S}}_{2, r}
\end{array}\right] .
$$

The matrix $\widehat{\mathcal{W}}$ is orthogonal of the same form as eq. 2.12 in the proof of lemma 1 with respect to eq. 2.14), and $\widehat{\mathcal{S}}_{i, r}=\operatorname{diag}\left(\sigma_{1}, \ldots, \sigma_{r}, 0, \ldots, 0\right) \in \mathbb{R}^{n_{i} \times n_{i}}$ where $\sigma_{i}$ are the singular values of $R_{1}^{-T} U_{1} S V_{2}^{T} R_{2}^{-1}$. Setting the choice $\widehat{R}^{-1}$ into eq. 2.3 and employing the fact that the 2-norm is invariant under unitary transformations, we obtain

$$
\operatorname{cond}\left(\widehat{R}^{-T} A \widehat{R}^{-1}\right)=\left\|\widehat{\mathcal{D}}^{-1 / 2} H \widehat{\mathcal{D}}^{-1 / 2}\right\|_{2}\left\|\widehat{\mathcal{D}}^{1 / 2} H^{-1} \widehat{\mathcal{D}}^{1 / 2}\right\|_{2},
$$

where $H$ is an SPD matrix given by

$$
H=\widehat{\mathcal{W}}^{T} \mathcal{W} \mathcal{D} \mathcal{W}^{T} \widehat{\mathcal{W}}, \quad \mathcal{D}=\left[\begin{array}{cc}
I_{1}+\mathcal{S}_{1, m} & 0 \\
0 & I_{2}-\mathcal{S}_{2, m}
\end{array}\right] .
$$

The matrices $\mathcal{W}$ eq. 2.12 and $\mathcal{S}_{i, m}$ are defined and constructed in the proof of lemma 1. Note that like $\widehat{\mathcal{W}}$, the matrix $\mathcal{W}$ is orthogonal. Hence, the product $\mathcal{W}^{T} \widehat{\mathcal{W}}$ is also an orthogonal matrix.

Our definitions so far indicate that the following diagonal matrices,

$$
\underline{\mathcal{D}}=\left[\begin{array}{cc}
I_{1} & 0 \\
0 & \left(I_{2}-\widehat{\mathcal{S}}_{2, r}\right)
\end{array}\right], \quad \overline{\mathcal{D}}=\left[\begin{array}{cc}
\left(I_{1}+\widehat{\mathcal{S}}_{1, r}\right) & 0 \\
0 & I_{2}
\end{array}\right] .
$$

bound the diagonal matrix $\widehat{\mathcal{D}}$

$$
\underline{\mathcal{D}} \leq \widehat{\mathcal{D}} \leq \overline{\mathcal{D}}
$$

in the sense that $(\widehat{\mathcal{D}}-\underline{\mathcal{D}})$ and $(\overline{\mathcal{D}}-\widehat{\mathcal{D}})$ are non-negative definite. Thus, applying the change of variables $\xi=\widetilde{\mathcal{D}}^{-1 / 2} x$ and exploiting the properties of the Rayleigh quotient, we can write

$$
\begin{aligned}
\left\|\widehat{\mathcal{D}}^{-1 / 2} H \widehat{\mathcal{D}}^{-1 / 2}\right\|_{2} & =\max _{x \neq 0} \frac{x^{T} \widehat{\mathcal{D}}^{-1 / 2} H \widehat{\mathcal{D}}^{-1 / 2} x}{x^{T} x}=\max _{\xi \neq 0} \frac{\xi^{T} H \xi}{\xi^{T} \widehat{\mathcal{D}} \xi} \\
& \geq \max _{\xi \neq 0} \frac{\xi^{T} H \xi}{\xi^{T} \overline{\mathcal{D}} \xi}=\max _{y \neq 0} \frac{y^{T} \overline{\mathcal{D}}^{-1 / 2} H \overline{\mathcal{D}}^{-1 / 2} y}{y^{T} y}=\left\|\overline{\mathcal{D}}^{-1 / 2} H \overline{\mathcal{D}}^{-1 / 2}\right\|_{2},
\end{aligned}
$$


where $y=\overline{\mathcal{D}}^{1 / 2} \xi$. Using the same arguments it can also be shown that

$$
\left\|\widehat{\mathcal{D}}^{1 / 2} H^{-1} \widehat{\mathcal{D}}^{1 / 2}\right\|_{2} \geq\left\|\underline{\mathcal{D}}^{1 / 2} H^{-1} \underline{\mathcal{D}}^{1 / 2}\right\|_{2} .
$$

Let $Z=\operatorname{span}\left\{e_{r+1}, \ldots, e_{n}\right\}$ where $e_{i}$ denotes the $i$-th canonical basis vector, and let $P_{Z}$ denote the orthogonal projection matrix on $Z$. The structure of $\overline{\mathcal{D}}$ implies

$$
\left\|\overline{\mathcal{D}}^{-1 / 2} H \overline{\mathcal{D}}^{-1 / 2}\right\|_{2} \geq \max _{P_{Z} y \neq 0} \frac{\left(P_{Z} y\right)^{T} \overline{\mathcal{D}}^{-1 / 2} H \overline{\mathcal{D}}^{-1 / 2} P_{Z} y}{\left(P_{Z} y\right)^{T} P_{Z} y}=\max _{P_{Z} y \neq 0} \frac{\left(P_{Z} y\right)^{T} H P_{Z} y}{\left(P_{Z} y\right)^{T} P_{Z} y} .
$$

Essentially, $P_{Z}^{T} H P_{Z}$ represents an $(n-r) \times(n-r)$ principal block of a unitarily equivalent matrix of $H$ whose eigenvalues are identical to $H$. Thus, by the Cauchy interlacing theorem eq. (2.11),

$$
\left\|\overline{\mathcal{D}}^{-1 / 2} H \overline{\mathcal{D}}^{-1 / 2}\right\|_{2} \geq \lambda_{r+1}(H)=1+\sigma_{r+1} .
$$

Applying similar arguments it can also be shown that

$$
\left\|\underline{\mathcal{D}}^{1 / 2} H^{-1} \underline{\mathcal{D}}^{1 / 2}\right\|_{2} \geq \lambda_{r+1}\left(H^{-1}\right)=\frac{1}{1-\sigma_{r+1}},
$$

which leads to the following lower bound on the spectral condition number,

$$
\operatorname{cond}\left(R^{-T} A R^{-1}\right) \geq \frac{1+\sigma_{r+1}}{1-\sigma_{r+1}} .
$$

Finally, let us consider the specific choice $U_{1} S V_{2}^{T}=R_{1}^{T} \mathcal{U}_{r} \Sigma \mathcal{V}_{r}^{T} R_{2}$ where $\mathcal{U}_{r}$ and $\mathcal{V}_{r}$ are composed of the first $r$ columns of $\mathcal{U}$ and $\mathcal{V}$, respectively, in the SVD of $R_{1}^{-T} M R_{2}^{-1}$. Consequently, we have $\sigma_{i}=\sigma_{i}, i=1, \ldots, r$. Thus, setting accordingly $\widehat{\mathcal{W}}=\mathcal{W}$ and $\widehat{R}=R$ we obtain by direct calculations:

$$
\operatorname{cond}\left(R^{-T} A R^{-1}\right)=\left\|R^{-T} A R^{-1}\right\|_{2}\left\|R A^{-1} R^{T}\right\|_{2}=\frac{1+\sigma_{r+1}}{1-\sigma_{r+1}}
$$

and the proof is complete.

\section{The Multilevel Weighted HODLR Preconditioning Scheme}

In this section we introduce the multilevel HODLR preconditioning scheme for SPD matrices. The method is based on the theory presented in section 2 and relaxation of the original problem. The motivation is twofold. First we demonstrate that the 1-level analysis can be extended to a multilevel preconditioning scheme. Second, we provide spectral bounds on the eigenvalues of the preconditioned system which give account for error propagation through the levels of the hierarchy.

In section 3.1 we give a brief review of the HODLR matrix structure which will be employed throughout the remainder of this paper. We focus on the symmetric case, since this work is concerned with the preconditioning of SPD matrices. In section 3.2 we introduce the preconditioning scheme, which is based on hierarchical construction and fast application of the inverse square roots, $R^{-1}$ and $R^{-T}$. In section 3.3 we briefly consider the associated memory and the computational costs of constructing and applying the scheme. An in-depth spectral analysis is presented in section 3.4. Our analysis provides estimates of the spectral bounds of the preconditioned system at each level, that take into account the approximation error at the lower levels of the hierarchy. These bounds are, however, of qualitative nature as they reflect a possible worst case scenario which is not likely to occur in practice. A rigorous and detailed proof of the theory is given in section 3.5 . 


\subsection{Symmetric HODLR Matrix Structure}

A symmetric HODLR matrix, $K \in \mathbb{R}^{n \times n}$, can be described in the following recursive manner,

$$
K=K_{1}^{(0)}, \quad K_{k}^{(\ell)}=\left[\begin{array}{cc}
K_{2 k-1}^{(\ell+1)} & U_{2 k-1}^{(\ell+1)} S_{k}^{(\ell)} V_{2 k}^{(\ell+1)^{T}} \\
V_{2 k}^{(\ell+1)} S_{k}^{(\ell)} U_{2 k-1}^{(\ell+1)^{T}} & K_{2 k}^{(\ell+1)}
\end{array}\right] \in \mathbb{R}^{n_{k}^{(\ell)} \times n_{k}^{(\ell)}},
$$

for $\ell=0,1, \ldots, L-1$ and $k=1,2, \ldots, 2^{\ell}$, where $\ell$ is the level of $K_{k}^{(\ell)}$ in the hierarchy. The triple products, $U_{2 k-1}^{(\ell+1)} S_{k}^{(\ell)} V_{2 k}^{(\ell+1)^{T}}$, represent low-rank blocks in the sense that

$$
U_{2 k-1}^{(\ell+1)} \in \mathbb{R}^{n_{2 k-1}^{(\ell+1)} \times r_{k}^{(\ell)}}, \quad V_{2 k}^{(\ell+1)} \in \mathbb{R}^{n_{2 k}^{(\ell+1)} \times r_{k}^{(\ell)}}, \quad S_{k}^{(\ell)} \in \mathbb{R}_{k}^{r_{k}^{(\ell)} \times r_{k}^{(\ell)}}
$$

where $r_{k}^{(\ell)}$ is the rank of the corresponding off-diagonal block of $K$. Typically, $r_{k}^{(\ell)} \ll n_{2 k-1}^{(\ell+1)}, n_{2 k}^{(\ell+1)}$. An illustration of the hierarchical structure of $K$ is displayed in fig. 2 .

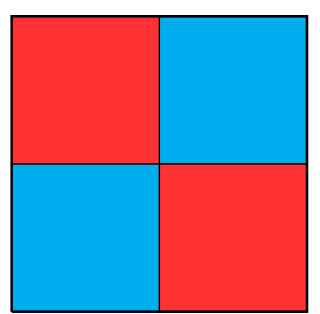

Level 1

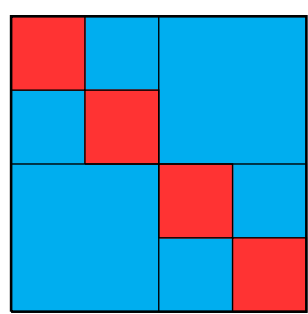

Level 2

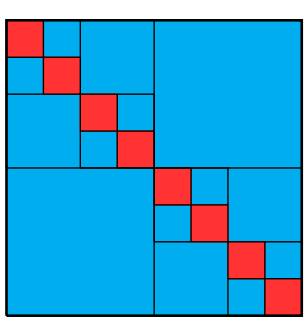

Level 3

Figure 2: The HODLR Structure. The first 3 levels, $\ell=1,2,3$, of the HODLR structure are illustrated: at each level the blue color blocks are the low-rank off-diagonal blocks and the red blocks are the HODLR principal submatrices.

The common practice is to set the HODLR matrix as an approximation of a given matrix, $A \in \mathbb{R}^{n \times n}$. The low-rank off-diagonal blocks of $K$ satisfy

$$
\left\|M_{k}^{(\ell)}-U_{2 k-1}^{(\ell+1)} S_{k}^{(\ell)} V_{2 k}^{(\ell+1)^{T}}\right\|_{2} \leq \tau_{k}^{(\ell)} \cdot\left\|M_{k}^{(\ell)}\right\|_{2}
$$

where $M_{k}^{(\ell)}$ denotes the corresponding off-diagonal block of $A$ and $\tau_{k}^{(\ell)}>0$ is a chosen tolerance. Typically, a prior reordering of the matrix rows and columns is needed to confirm that $r_{k}^{(\ell)}$ are, indeed, low.

For obtaining low-rank approximations satisfying eq. (3.3), the low-rank singular value decomposition (SVD) [16] which originated in [1] is, generally, considered the best choice, since it is optimal with respect to any unitarily invariant norm (2-norm, Frobenius). The computational cost required to obtain the SVD of $M_{k}^{(\ell)}$ is relatively expensive necessitating an $\mathcal{O}\left(\mathrm{m}^{3}\right)$ operations, where $m=n_{k}^{(\ell)} / 2$. For this reason a variety of fast approximation algorithms attempting to efficiently obtain a low-rank approximation close enough to the low-rank SVD have been proposed. These include, among others, the rank revealing LU [27], rank revealing QR [18], randomized algorithms [13, 25, 34, adaptive cross approximation [30] and boundary distance low-rank [2]. For more details see a review on this topic in [2]. 


\subsection{Recursive Formula of the Multilevel Preconditioning Scheme}

By our definitions eq. (3.1) the principal blocks of $K$ and the corresponding blocks of $A$ are described by

$$
K_{k}^{(\ell)}=\left[\begin{array}{cc}
K_{2 k-1}^{(\ell+1)} & U_{2 k-1}^{(\ell+1)} S_{k}^{(\ell)} V_{2 k}^{(\ell+1)^{T}} \\
V_{2 k}^{(\ell+1)} S_{k}^{(\ell)} U_{2 k-1}^{(\ell+1)^{T}} & K_{2 k}^{(\ell+1)}
\end{array}\right], A_{k}^{(\ell)}=\left[\begin{array}{cc}
A_{2 k-1}^{(\ell+1)} & M_{k}^{(\ell)} \\
M_{k}^{(\ell)^{T}} & A_{2 k}^{(\ell+1)}
\end{array}\right],
$$

where $K_{k}^{(\ell)}, A_{k}^{(\ell)} \in \mathbb{R}^{n_{k}^{(\ell)} \times n_{k}^{(\ell)}}$, and the rank of each off-diagonal triple product approximation satisfies

$$
\operatorname{rk}\left(U_{2 k-1}^{(\ell+1)} S_{k}^{(\ell)} V_{2 k}^{(\ell+1)^{T}}\right) \leq r_{k}^{(\ell)} .
$$

The key idea we propose is to construct each $K_{k}^{(\ell)}$ as an optimal 1-level preconditioning scheme of the matrix

$$
B_{k}^{(\ell)}=\left[\begin{array}{cc}
K_{2 k-1}^{(\ell+1)} & M_{k}^{(\ell)} \\
M_{k}^{(\ell)^{T}} & K_{2 k}^{(\ell+1)}
\end{array}\right] \in \mathbb{R}^{n_{k}^{(\ell)} \times n_{k}^{(\ell)}}
$$

which is obtained by replacing the principal blocks of $A_{k}^{(\ell)}$ with the corresponding principal blocks of $K_{k}^{(\ell)}$. This is a relaxation of the original problem, which facilitates a fast construction method. The resulting preconditioned global system condition number is no longer ensured to be minimal. However, the numerical results in section 4 indicate that the proposed approach is highly robust and, in general, attains superior condition number compared to HODLR approximations using other low-rank approximation schemes.

Before proceeding we introduce some necessary notations. Let $R_{k}^{(\ell)} \in \mathbb{R}^{n_{k}^{(\ell)} \times n_{k}^{(\ell)}}$ denote the square root of $K_{k}^{(\ell)}$ in the sense that $K_{k}^{(\ell)}=R_{k}^{(\ell)^{T}} R_{k}^{(\ell)}$. Let $\mathcal{U}_{k, r}^{(\ell)} \in \mathbb{R}^{n_{2 k-1}^{(\ell+1)} \times r_{k}^{(\ell)}}$ and $\mathcal{V}_{k, r}^{(\ell)} \in$ $\mathbb{R}^{n_{2 k}^{(\ell+1)} \times r_{k}^{(\ell)}}$ be two thin matrices with orthogonal columns composed of the first $r_{k}^{(\ell)}$ left and right, respectively, singular vectors of the SVD,

$$
R_{2 k-1}^{(\ell+1)^{-T}} M_{k}^{(\ell)} R_{2 k}^{(\ell+1)^{-1}}=\mathcal{U}_{k}^{(\ell)} \Sigma_{k}^{(\ell)} \mathcal{V}_{k}^{(\ell)^{T}}
$$

where $\Sigma_{k, r}^{(\ell)} \in \mathbb{R}_{k}^{r_{k}^{(\ell)} \times r_{k}^{(\ell)}}$ is the principal submatrix of

$$
\Sigma_{k}^{(\ell)}=\operatorname{diag}\left(\sigma_{k, 1}^{(\ell)}, \ldots, \sigma_{k, \min \left\{n_{2 k-1}^{(\ell+1)}, n_{2 k}^{(\ell+1)}\right\}}^{(\ell)}\right)
$$

For brevity and clarity we will abuse the notation and employ $\mathcal{U}=\mathcal{U}_{k, r}^{(\ell)}$ and $\mathcal{V}=\mathcal{V}_{k, r}^{(\ell)}$. The proof of theorem 1 implies that the following recursive formulas for a fast application of the inverse square roots hold,

$$
R_{k}^{(\ell)^{-T}}=\left(I+\frac{1}{2}\left[\begin{array}{cc}
\mathcal{U}_{r} & \mathcal{U}_{r} \\
\mathcal{V}_{r} & -\mathcal{V}_{r}
\end{array}\right]\left[\begin{array}{cc}
\mathcal{S}_{r}^{+}-I & 0 \\
0 & \mathcal{S}_{r}^{-}-I
\end{array}\right]\left[\begin{array}{cc}
\mathcal{U}_{r}^{T} & \mathcal{V}_{r}^{T} \\
\mathcal{U}_{r}^{T} & -\mathcal{V}_{r}^{T}
\end{array}\right]\right)\left[\begin{array}{cc}
R_{2 k-1}^{(\ell+1)} & 0 \\
0 & R_{2 k}^{(\ell+1)}
\end{array}\right]^{-T}
$$

and

$$
R_{k}^{(\ell)^{-1}}=\left[\begin{array}{cc}
R_{2 k-1}^{(\ell+1)} & 0 \\
0 & R_{2 k}^{(\ell+1)}
\end{array}\right]^{-1}\left(I+\frac{1}{2}\left[\begin{array}{cc}
\mathcal{U}_{r} & \mathcal{U}_{r} \\
\mathcal{V}_{r} & -\mathcal{V}_{r}
\end{array}\right]\left[\begin{array}{cc}
\mathcal{S}_{r}^{+}-I & 0 \\
0 & \mathcal{S}_{r}^{-}-I
\end{array}\right]\left[\begin{array}{cc}
\mathcal{U}_{r}^{T} & \mathcal{V}_{r}^{T} \\
\mathcal{U}_{r}^{T} & -\mathcal{V}_{r}^{T}
\end{array}\right]\right)
$$

where $\mathcal{S}_{r}^{ \pm}=\left(I \pm \Sigma_{k, r}^{(\ell)}\right)^{-\frac{1}{2}}$. These formulas can be verified by writing the product $R_{k}^{(\ell)^{-T}} K_{k}^{(\ell)} R_{k}^{(\ell)^{-1}}$ which, indeed, equals to the identity matrix, assuming $K_{k}^{(\ell)}$ is SPD. 


\subsection{Utilization and Construction of the Preconditioning Scheme}

The recursive representations of $R_{k}^{(\ell)^{-1}}$ and $R_{k}^{(\ell)^{-T}}$ indicate that both matrices, essentially, posses HODLR structure. Thus, $R_{k}^{(\ell)^{-1}}$ and $R_{k}^{(\ell)^{-T}}$ can be applied relatively fast in matrix product operations. In the case that a constant average rank, $r^{(\ell)}=\mathcal{O}(r)$, is taken for the off-diagonal blocks, the recursive representations implies the following relation

$$
\mathcal{C}^{(\ell)}(m, n, r)=2 \mathcal{C}^{(\ell+1)}(m, n, r)+\mathcal{O}\left(m \cdot \frac{n}{2^{\ell}} \cdot r\right),
$$

where $\mathcal{C}^{(\ell)}(m, n, r)$ denotes the computational cost of the operation $R_{k}^{(\ell)^{-T}} \cdot F_{k}^{(\ell)}$ at level $\ell$, $F_{k}^{(\ell)} \in \mathbb{R}^{n_{k}^{(\ell)} \times m}$, and $n_{k}^{(\ell)}=n^{(\ell)}=n / 2^{\ell}$ is assumed. The first contribution in eq. 3.7 stems from the recursive calls of the inverse square roots of the diagonal blocks. The second contribution is associated with the cost of the matrix product operations. Expanding eq. (3.7) into a sum yields the total computational cost estimate,

$$
\mathcal{C}^{(0)}(m, n, r)=\sum_{\ell=0}^{\log \left(\frac{n}{r}\right)} \mathcal{O}(m \cdot r n)=\mathcal{O}(m \cdot r n \log (n))
$$

where the depth of the hierarchy is set by $n=2^{L} r$. Similarly, the cost of storing $R_{k}^{(0)^{-1}}$ and $R_{k}^{(0)^{-T}}$ is equal to $\mathcal{O}(r n \log n)$ in the case where the average rank per level, $r^{(\ell)}$, is of $\mathcal{O}(r)$. See [2] for further details. As noted in the introduction if the HODLR scheme is generalized to HSS the costs to apply the preconditioner reduce to about $\mathcal{O}(n)$, see [37] for further details.

Constructing the preconditioner is accomplished by performing a single pass over the hierarchy from bottom to top. At each level $\ell$ we compute the low-rank factorizations of the triple products

$$
R_{2 k-1}^{(\ell+1)^{-T}} M_{k}^{(\ell)} R_{2 k}^{(\ell+1)^{-1}}
$$

where $k=1,2, \ldots, 2^{\ell}$. Obtaining the low-rank factorization is performed by the following procedure:

- Capture the range of eq. 33.8 in a matrix $Q_{L}^{(k, \ell)}$ whose columns are orthonormal,

$$
\left(I-Q_{L}^{(k, \ell)} Q_{L}^{(k, \ell)^{T}}\right) \cdot R_{2 k-1}^{(\ell+1)^{-T}} M_{k}^{(\ell)} R_{2 k}^{(\ell+1)^{-1}} \approx \mathbf{0}, \quad Q_{L}^{(k, \ell)^{T}} Q_{L}^{(k, \ell)}=I .
$$

- Capture the range of the transpose of eq. 3.8 in a matrix $Q_{R}^{(k, \ell)}$ whose columns are orthonormal,

$$
\left(I-Q_{R}^{(k, \ell)} Q_{R}^{(k, \ell)^{T}}\right) \cdot R_{2 k}^{(\ell+1)^{-T}} M_{k}^{(\ell)^{T}} R_{2 k-1}^{(\ell+1)^{-1}} \approx \mathbf{0}, \quad Q_{R}^{(k, \ell)^{T}} Q_{R}^{(k, \ell)}=I .
$$

- Compute the rank $r^{(k, \ell)}$ truncated SVD of the reduced matrix,

$$
Q_{L}^{(k, \ell)^{T}} \cdot R_{2 k-1}^{(\ell+1)^{-T}} M_{k}^{(\ell)} R_{2 k}^{(\ell+1)^{-1}} \cdot Q_{R}^{(k, \ell)} \approx U_{k, r}^{(\ell)} \Sigma_{k, r}^{(\ell)} V_{k, r}^{(\ell)}
$$

- Reconstruct the WSVD left and right singular vectors matrices,

$$
\mathcal{U}_{k, r}^{(\ell)}=Q_{L} \cdot U_{k, r}, \quad \mathcal{V}_{k, r}^{(\ell)}=Q_{R} \cdot V_{k, r}
$$

If the effective rank of eq. 3.8 is small, e.g. $\mathcal{O}(1)$, we can capture the range matrices, $Q_{L}^{(k, \ell)}$ and $Q_{R}^{(k, \ell)}$, quickly by applying eq. (3.8) and its traspose on a small set of randomized column vectors. See [26] for more details. However, if the effective rank of eq. 3.8 is not small, the procedure can become costly. 


\subsection{Spectral Estimates and Error Propagation}

Let us now focus on the spectral properties of the preconditioned submatrices, $R_{k}^{(\ell)^{-T}} A_{k}^{(\ell)} R_{k}^{(\ell)^{-1}}$, where $R_{k}^{(\ell)}$ is the square root of the principal submatrix $K_{k}^{(\ell)}$. The submatrix $A_{k}^{(\ell)}$ is given in eq. (3.4). Clearly the important case is $\ell=0$, since we are ultimately interested in preconditioning the input matrix, $A=A_{1}^{(0)}$.

For brevity and clarity we will abuse the notation and employ the following representations in the spirit of section 2 .

$$
K_{k}^{(\ell)}=\left[\begin{array}{cc}
K_{1} & U_{1} S V_{2}^{T} \\
V_{2} S U_{1}^{T} & K_{2}
\end{array}\right], \quad A_{k}^{(\ell)}=\left[\begin{array}{cc}
A_{1} & M \\
M^{T} & A_{2}
\end{array}\right], \quad R_{i}^{T} R_{i}=K_{i}(i=1,2) .
$$

Note that $R_{i}$ represents an approximate square root of $A_{i}$, as opposed to the exact square root that was assumed in section 2. We make the fundamental assumption that we have at our disposal spectral bounds estimates,

$$
\alpha_{i} \leq \lambda_{\min }\left(R_{i}^{-T} A_{i} R_{i}^{-1}\right) \leq \lambda_{\max }\left(R_{i}^{-T} A_{i} R_{i}^{-1}\right) \leq \beta_{i} \quad(i=1,2) .
$$

The lower-level bounds, $\alpha_{i}$ and $\beta_{i}(i=1,2)$, can be obtained numerically, or possibly estimated analytically by the theory presented in this subsection. Note that in the case $\ell=L-1$ we have $\alpha_{i}=1=\beta_{i}$, and in the case $\ell=L-2$ we have from section 2 the exact bounds

$$
\begin{aligned}
& \alpha_{i}=\lambda_{\min }\left(R_{i}^{-T} A_{i} R_{i}^{-1}\right)=1-\sigma_{2 k-2+i, r}^{(L-1)} \in(0,1], \\
& \beta_{i}=\lambda_{\max }\left(R_{i}^{-T} A_{i} R_{i}^{-1}\right)=1+\sigma_{2 k-2+i, r}^{(L-1)} \in[1,2) .
\end{aligned}
$$

The main result of the current subsection is presented in theorem 2 . The theorem provides a description of the behavior of the current-level spectral bounds,

$$
\alpha \leq \lambda_{\min }\left(R_{k}^{(\ell)^{-T}} A_{k}^{(\ell)} R_{k}^{(\ell)^{-1}}\right) \leq \lambda_{\max }\left(R_{k}^{(\ell)^{-T}} A_{k}^{(\ell)} R_{k}^{(\ell)^{-1}}\right) \leq \beta,
$$

as a function of the lower-level bounds eq. $\sqrt{3.9}$ and the rank of the off-diagonal blocks, $r=r_{k}^{(\ell)}$. The definition of the bounds $\alpha$ and $\beta$ eq. $(3.10)$ is based on variational formulation and provided in lemma 2 The analysis requires sufficient (but not necessary) conditions on the given lowerlevel bounds, $\alpha_{i}$ and $\beta_{i}(i=1,2)$. We show that the proposed HODLR preconditioning scheme, essentially, maps both the $r$ largest and the $r$ smallest eigenvalues to a closed segment containing 1. When this segment is small, the preconditioner retains optimality or near optimality. We also show that the sensitivity of the spectral bounds to the inaccuracies $K_{i} \neq A_{i}(i=1,2)$ is governed by the Cauchy-Bunyakowski-Schwarz (CBS) constant [3, 4].

Theorem 2. Let

$$
A=\left[\begin{array}{cc}
A_{1} & M \\
M^{T} & A_{2}
\end{array}\right], \quad K=\left[\begin{array}{cc}
K_{1} & U_{1} S V_{2}^{T} \\
V_{2} S U_{1}^{T} & K_{2}
\end{array}\right]
$$

be symmetric matrices of the same dimensions and partition where $A$ is SPD. Assume that the off-diagonal triple product approximation $U_{1} S V_{2}^{T}$ satisfy

$$
U_{1}=R_{1}^{T} \mathcal{U}_{r}, \quad S=\Sigma_{r}, \quad V_{2}=R_{2}^{T} \mathcal{V}_{r}
$$

where $\mathcal{U}_{r}$ and $\mathcal{V}_{r}$ are composed of the first $r$ left and right, respectively, singular vectors of the $S V D$

$$
R_{1}^{-T} M R_{2}^{-1}=\mathcal{U} \Sigma \mathcal{V}^{T}, \quad \Sigma=\operatorname{diag}\left(\sigma_{1}, \ldots, \sigma_{\min \left\{n_{1}, n_{2}\right\}}\right)
$$


and $R_{i}^{T} R_{i}=K_{i}(i=1,2)$.

Assuming there exist real positive constants,

$$
0<\alpha_{1}, \alpha_{2} \leq 1 \leq \beta_{1}, \beta_{2},
$$

such that

$$
0<\alpha_{i} x_{i}^{T} K_{i} x_{i} \leq x_{i}^{T} A_{i} x_{i} \leq \beta_{i} x_{i}^{T} K_{i} x_{i} \quad \forall x_{i} \in \mathbb{R}^{n_{i}},
$$

we have the following spectral estimates:

1. If $\sigma_{1}<\sqrt{\alpha_{1} \alpha_{2}}$ then

$$
\alpha=\min \left\{\alpha_{1,2}^{\mathrm{avg}}-\sqrt{\sigma_{r+1}^{2}+\left(\alpha_{1,2}^{\text {dif }}\right)^{2}} \quad, \quad \frac{\alpha_{1,2}^{\mathrm{avg}}+\sigma_{1}}{1+\sigma_{1}}-\delta_{\alpha}^{1,2}\right\},
$$

where

$$
\alpha_{1,2}^{\mathrm{avg}}=\frac{\alpha_{1}+\alpha_{2}}{2}, \quad \alpha_{1,2}^{\mathrm{dif}}=\frac{\alpha_{1}-\alpha_{2}}{2}, \quad(\alpha=\alpha \text { or } \beta),
$$

and

$$
\delta_{\alpha}^{1,2}=\sqrt{\frac{1}{4}\left|\frac{\alpha_{1,2}^{\mathrm{avg}}-\sigma_{1}}{1-\sigma_{1}}-\frac{\alpha_{1,2}^{\mathrm{avg}}+\sigma_{1}}{1+\sigma_{1}}\right|^{2}+\frac{\left(\alpha_{1,2}^{\mathrm{dif}}\right)^{2}}{1-\sigma_{1}^{2}}}-\frac{1}{2}\left|\frac{\alpha_{1,2}^{\mathrm{avg}}-\sigma_{1}}{1-\sigma_{1}}-\frac{\alpha_{1,2}^{\mathrm{avg}}+\sigma_{1}}{1+\sigma_{1}}\right|,
$$

is a positive lower spectral bound of the preconditioned system,

$$
0<\alpha \leq \lambda\left(R^{-T} A R^{-1}\right), \quad K=R^{T} R .
$$

2. If $\sigma_{1}<1$ then

$$
\beta=\max \left\{\beta_{1,2}^{\text {avg }}+\sqrt{\sigma_{r+1}^{2}+\left(\beta_{1,2}^{\text {dif }}\right)^{2}} \quad, \quad \frac{\beta_{1,2}^{\text {avg }}-\sigma_{1}}{1-\sigma_{1}}+\delta_{\beta}^{1,2}\right\}
$$

where

$$
\beta_{1,2}^{\mathrm{avg}}=\frac{\beta_{1}+\beta_{2}}{2}, \quad \beta_{1,2}^{\mathrm{dif}}=\frac{\beta_{1}-\beta_{2}}{2}, \quad(\beta=\alpha \text { or } \beta),
$$

and

$$
\delta_{\beta}^{1,2}=\sqrt{\frac{1}{4}\left|\frac{\beta_{1,2}^{\mathrm{avg}}-\sigma_{1}}{1-\sigma_{1}}-\frac{\beta_{1,2}^{\mathrm{avg}}+\sigma_{1}}{1+\sigma_{1}}\right|^{2}+\frac{\left(\beta_{1,2}^{\mathrm{dif}}\right)^{2}}{1-\sigma_{1}^{2}}}-\frac{1}{2}\left|\frac{\beta_{1,2}^{\mathrm{avg}}-\sigma_{1}}{1-\sigma_{1}}-\frac{\beta_{1,2}^{\mathrm{avg}}+\sigma_{1}}{1+\sigma_{1}}\right| .
$$

is an upper spectral bound of the preconditioned system,

$$
0<\lambda\left(R^{-T} A R^{-1}\right) \leq \beta, \quad K=R^{T} R .
$$

Remarks 1. The justification for eq. (3.11) is a consequence of theorem 2, which shows that $\alpha$ eq. (3.13) and $\beta$ eq. (3.14) are monotonically non-increasing and non-decreasing as functions of the level, respectively. This observation is also supported by numerical evidence in section 4 . If $\sqrt{\alpha_{1} \alpha_{2}} \leq \sigma_{1}<1$, then the preconditioned system remains SPD. However, the theory presented here can not predict the positive value of the lower spectral bound, $\alpha$ eq. (3.13). 


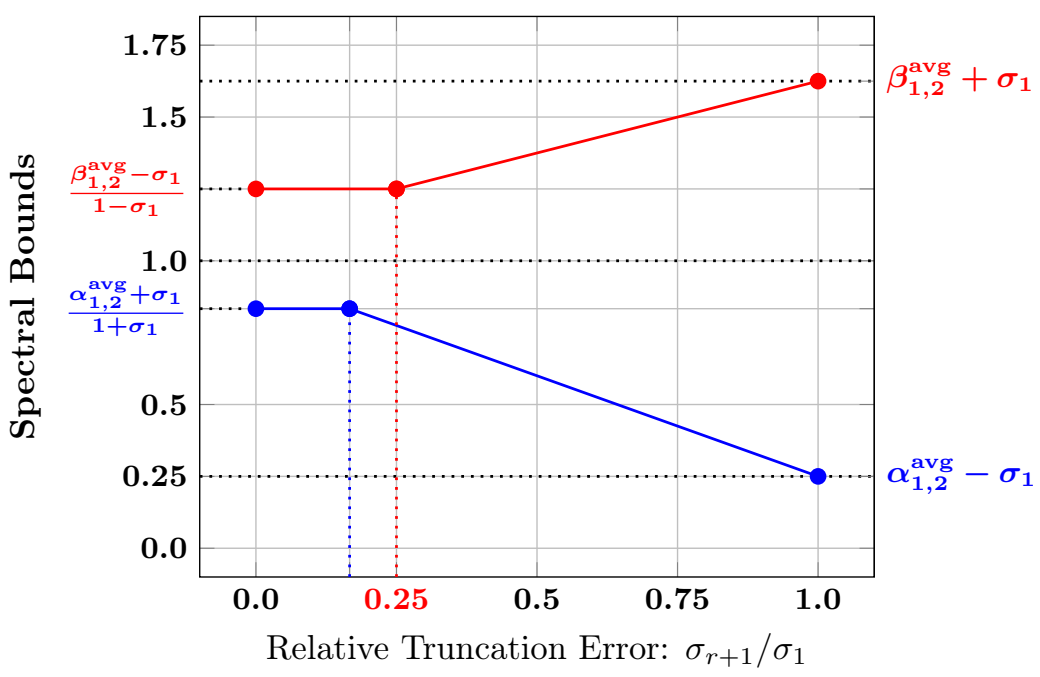

Figure 3: Spectral Bounds. A typical behavior of the spectral bounds displayed for the case $\alpha_{1}=\alpha_{2}$ and $\beta_{1}=\beta_{2}$. The lower bound $\alpha$ eq. 3.13 vs. $\sigma_{r+1} / \sigma_{1}$ is plotted in blue, and the upper bound $\beta$ eq. 3.14 vs. $\sigma_{r+1} / \sigma_{1}$ is plotted in red.

From theorem 2 we observe that each estimated bound, $\alpha$ or $\beta$, is a minimum or a maximum, respectively, of two competing terms: the first depends on the largest singular value, $\sigma_{1}$, and the second is a function of the truncation error, $\sigma_{r+1}$. In fact, when the truncation error becomes sufficiently small it does not affect the values of the bounds, which are governed solely by the terms depending on the largest singular value. Thus in this case, improving the approximation by increasing the rank $r$ does not improve the corresponding condition number estimate, $\beta / \alpha$. An illustration of this observation is given in fig. 3 .

The last observation as displayed in fig. 3indicates that the value of $\sigma_{1}$ is central to the estimation of the spectral bounds, and effectively dominates the condition number of the preconditioned system. In this sense $\sigma_{1}$ reflects the sensitivity of the condition number of $R_{k}^{(\ell)^{-T}} A_{k}^{(\ell)} R_{k}^{(\ell)^{-1}}$ to the lower level inaccuracies. It can be shown that $\sigma_{1}$ is the so-called Cauchy-Bunyakowski-Schwarz (CBS) constants of the matrix $K$, which is defined by

$$
\sigma_{1}=\sup _{x_{1}, x_{2} \neq 0} \frac{x_{1}^{T} M x_{2}}{\sqrt{x_{1}^{T} K_{1} x_{1}} \sqrt{x_{2}^{T} K_{2} x_{2}}} \geq 0 .
$$

Definition eq. 3.15) originated from the theory of Algebraic Multilevel Iterations Methods [3, 4], and coincides with the principal angle (cosine of the smallest angle) between the column space of $\left[\begin{array}{ll}I_{1} & 0\end{array}\right]^{T}$ and the column space of $\left[\begin{array}{ll}0 & I_{2}\end{array}\right]^{T}$ with respect to the inner product $\langle x, y\rangle_{A}=y^{T} A x$. Thus, $\sigma_{1}$ represents the local contribution of the upper level to the overall condition number. Using eq. (3.15) with assumption eq. (3.11) leads to the following relation

$$
\frac{1}{\sqrt{\beta_{1} \beta_{2}}} \leq \frac{\sigma_{1}}{\sigma_{1}^{\text {exact }}} \leq \frac{1}{\sqrt{\alpha_{1} \alpha_{2}}}
$$

where $\sigma_{1}^{\text {exact }}$ is the corresponding CBS constant of $A$. The important conclusion here is that $\sigma_{1}$ and $\sigma_{1}^{\text {exact }}$ are correlated where $\sigma_{1}^{\text {exact }}$ is intrinsically predetermined by the given matrix, $A$, and the chosen partition. If $K$ is close to $A$ then we can expect $\sigma_{1}$ to be close to $\sigma_{1}^{\text {exact }}$, and in this case we have little influence over its value. 
Regarding the spectrum of the preconditioned system, the interpretation of theorem 2 is similar to the interpretation of theorem 1. From the proof it can be inferred that two-sided block Jacobi (i.e., the case $r=0$ ) effectively maps the spectra of the bounding preconditioned systems to two segments centered around $\alpha_{1,2}^{\text {avg }}$ and $\beta_{1,2}^{\text {avg }}$,

$$
\begin{aligned}
& {\left[\alpha_{1,2}^{\mathrm{avg}}-\sqrt{\sigma_{1}^{2}+\left(\alpha_{1,2}^{\mathrm{dif}}\right)^{2}}, \alpha_{1,2}^{\mathrm{avg}}+\sqrt{\sigma_{1}^{2}+\left(\alpha_{1,2}^{\text {dif }}\right)^{2}}\right],} \\
& {\left[\beta_{1,2}^{\mathrm{avg}}-\sqrt{\sigma_{1}^{2}+\left(\beta_{1,2}^{\text {dif }}\right)^{2}}, \beta_{1,2}^{\mathrm{avg}}+\sqrt{\sigma_{1}^{2}+\left(\beta_{1,2}^{\text {dif }}\right)^{2}}\right] .}
\end{aligned}
$$

The multilevel Weighted HODLR preconditioning scheme does the same, but also maps the largest and smallest eigenvalues to the segments

$$
\begin{aligned}
& {\left[\frac{\sigma_{1}+\alpha_{1,2}^{\mathrm{avg}}}{1+\sigma_{1}}-\delta_{\alpha}^{1,2}, \frac{\sigma_{1}-\alpha_{1,2}^{\mathrm{avg}}}{1-\sigma_{1}}+\delta_{\alpha}^{1,2}\right],} \\
& {\left[\frac{\beta_{1,2}^{\mathrm{avg}}+\sigma_{1}}{1+\sigma_{1}}-\delta_{\beta}^{1,2}, \frac{\beta_{1,2}^{\mathrm{avg}}-\sigma_{1}}{1-\sigma_{1}}+\delta_{\beta}^{1,2}\right],}
\end{aligned}
$$

respectively. Thus, assuming the segments eqs. (3.16) and 3.17 are small, a significant improvement in the condition number as well as the clustering of the spectrum of the original preconditioned system is expected. An illustration is given in fig. 4. The figure is similar to fig. 1 where the main difference is that the weighted HODLR preconditioning scheme now maps the extreme eigenvalues to an interval containing 1 and not exactly to 1 .

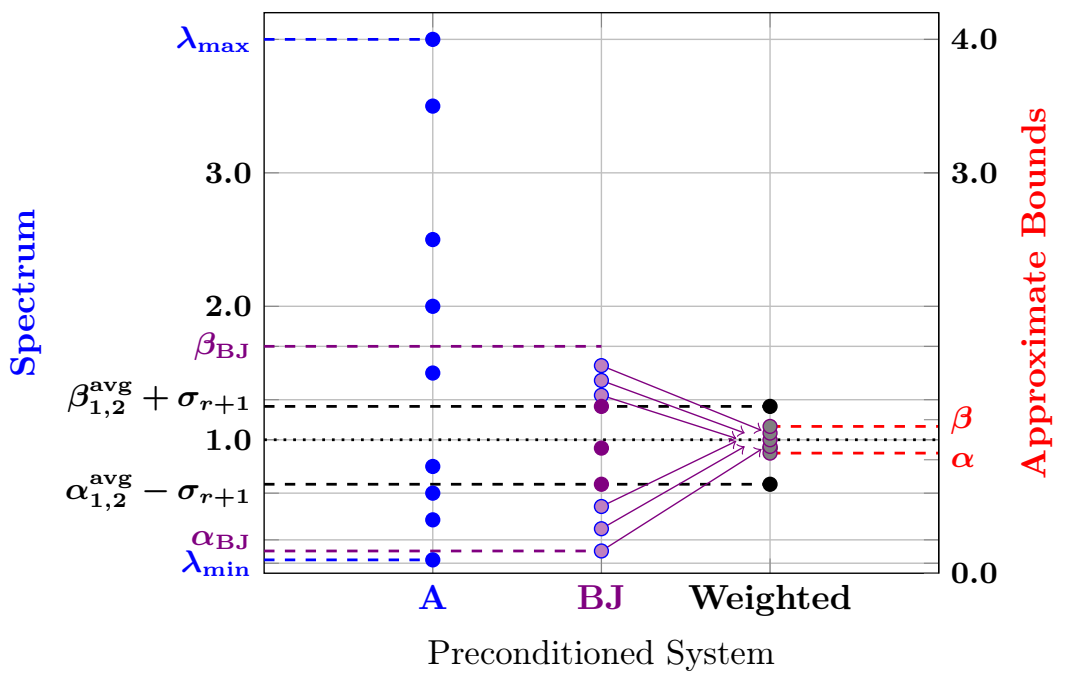

Figure 4: Spectrum Clustering for the Multilevel Weighted HODLR Preconditioning Scheme. The spectrum of some SPD matrix $A$ and the transformation it goes after preconditioning by block Jacobi (BJ) and the multilevel weighted HODLR preconditioning scheme are displayed. The spectra are ordered from the left to the right starting from $A$, followed by Block Jacobi (BJ) and ends up with the multilevel weighted HODLR scheme. The spectral bounds $\alpha$ eq. (3.13) and $\beta$ eq. (3.14) are marked on the right $y$-axis, while the spectral bounds for the block Jacobi case $\alpha_{\mathrm{BJ}}=\alpha(r=0)$ and $\beta_{\mathrm{BJ}}=\beta(r=0)$ are marked on the left $y$-axis. 


\subsection{Proof of Theorem 2}

The proof of theorem 2 is based on lemma 2 which provides the definition of the bounds $\alpha$ eq. (3.13) and $\beta$ eq. (3.14), and on the technical result presented in lemma 3

Lemma 2. Let

$$
A=\left[\begin{array}{cc}
A_{1} & M \\
M^{T} & A_{2}
\end{array}\right], \quad K=\left[\begin{array}{cc}
K_{1} & U_{1} S V_{2}^{T} \\
V_{2} S U_{1}^{T} & K_{2}
\end{array}\right],
$$

and the associated lower-level bounds

$$
0<\alpha_{1}, \alpha_{2} \leq 1 \leq \beta_{1}, \beta_{2}
$$

satisfy the assumptions of theorem 2

Let us define

$$
\underline{K}=\left[\begin{array}{cc}
\alpha_{1} K_{1} & M \\
M^{T} & \alpha_{2} K_{2}
\end{array}\right], \quad \bar{K}=\left[\begin{array}{cc}
\beta_{1} K_{1} & M \\
M^{T} & \beta_{2} K_{2}
\end{array}\right] .
$$

Then we have:

1. The matrices $\underline{K}, \bar{K}$ are $S P D$ iff

$$
\sigma_{1}<\sqrt{\alpha_{1} \alpha_{2}}, \quad \sigma_{1}<\sqrt{\beta_{1} \beta_{2}},
$$

respectively, where $\sigma_{1}$ is the largest singular of $R_{1}^{-T} M R_{2}^{-1}$.

2. If $\underline{K}, \bar{K}$ are $S P D$, there exist two positive constants, $\alpha$ and $\beta$, such that

$$
\alpha=\min _{x \neq 0} \frac{x^{T} \underline{K} x}{x^{T} K x} \leq \lambda_{\min }\left(\widehat{B}^{T} A \widehat{B}\right) \leq \lambda_{\max }\left(\widehat{B}^{T} A \widehat{B}\right) \leq \max _{x \neq 0} \frac{x^{T} \bar{K} x}{x^{T} K x}=\beta .
$$

Proof. of lemma 2

To show the first part of the lemma we consider a general matrix of the form

$$
H=\left[\begin{array}{cc}
\delta_{1} K_{1} & M \\
M^{T} & \delta_{2} K_{2}
\end{array}\right], \quad \delta_{1}, \delta_{2}>0
$$

Let us apply the following two-sided transformations

$$
\widehat{H}=\left[\begin{array}{cc}
\frac{1}{\sqrt{\delta_{1}}} R_{1}^{-T} & 0 \\
0 & \frac{1}{\sqrt{\delta_{2}}} R_{2}^{-T}
\end{array}\right] H\left[\begin{array}{cc}
\frac{1}{\sqrt{\delta_{1}}} R_{1}^{-1} & 0 \\
0 & \frac{1}{\sqrt{\delta_{2}}} R_{2}^{-1}
\end{array}\right]=\left[\begin{array}{cc}
I & \frac{1}{\sqrt{\delta_{1} \delta_{2}}} \mathcal{M} \\
\frac{1}{\sqrt{\delta_{1} \delta_{2}}} \mathcal{M}^{T} & I
\end{array}\right]
$$

where $\mathcal{M}=R_{1}^{-T} M R_{2}^{-1}$. The matrix $\widehat{H}$ is SPD iff $H$ is SPD as well. Thus, by lemma 1 the matrix $H$ is SPD iff $1-\sigma_{1} / \sqrt{\delta_{1} \delta_{2}}>0$, and the conditions ensuring $\underline{K}, K$, and $\bar{K}$ are SPD immediately follow.

For the second part of the lemma it is sufficient to assume that $\underline{K}$ is SPD which, by the first part, ensures that $K$ and $\bar{K}$ are SPD as well. Accordingly, we obtain the following inequalities

$$
\frac{x^{T} K x}{x^{T} \underline{K} x} \leq \frac{x^{T} K x}{x^{T} A x} \leq \frac{x^{T} K x}{x^{T} \bar{K} x} \quad \forall x \neq 0 .
$$

The Lagrangian stationary points of each generalized Rayleigh quotient in the inequalities above constitute the spectrum of each preconditioned system. Thus, the proof is complete.

$\mathrm{RR} \mathrm{n}^{\circ} 9200$ 
Lemma 3. Let $H=\left[\begin{array}{ll}\mathcal{D}^{(1)} & \mathcal{D}^{(2)} \\ \mathcal{D}^{(2)} & \mathcal{D}^{(3)}\end{array}\right] \in \mathbb{R}^{2 r \times 2 r}$, where $\mathcal{D}^{(i)}(i=1,2,3)$ are diagonal matrices,

$$
\mathcal{D}^{(i)}=\operatorname{diag}\left(d_{1}^{(i)}, \ldots, d_{r}^{(i)}\right)
$$

If $d_{j}^{(2)} \neq 0$ for all $j=1,2, \ldots, r$, then

$$
\operatorname{spec}(H)=\left\{\lambda_{j}^{-}\right\}_{j=1}^{m} \cup\left\{\lambda_{j}^{+}\right\}_{j=1}^{m}, \quad \lambda_{j}^{ \pm}=\frac{d_{j}^{(1)}+d_{j}^{(3)}}{2} \pm \sqrt{\left(\frac{d_{j}^{(1)}-d_{j}^{(3)}}{2}\right)^{2}+\left(d_{j}^{(2)}\right)^{2}},
$$

where $\operatorname{spec}(H)$ denotes the spectrum of the symmetric matrix $H$.

Proof. of lemma 3 .

From the given structure of $H$ it is clear that $\lambda \in \mathbb{R}$ is an eigenvalue of $H$ iff for some $j=$ $1,2, \ldots, m$ the vectors $\left(d_{j}^{(1)}-\lambda, d_{j}^{(2)}\right)$ and $\left(d_{j}^{(2)}, d_{j}^{(3)}-\lambda\right)$ are linearly dependent. Since we have assumed $d_{j}^{(2)} \neq 0$, we have that $\left(d_{j}^{(1)}-\lambda, d_{j}^{(2)}\right)$ and $\left(d_{j}^{(2)}, d_{j}^{(3)}-\lambda\right)$ are linearly dependent iff

$$
\frac{d_{j}^{(1)}-\lambda}{d_{j}^{(2)}}=\frac{d_{j}^{(2)}}{d_{j}^{(3)}-\lambda} \Leftrightarrow\left(d_{j}^{(1)}-\lambda\right)\left(d_{j}^{(3)}-\lambda\right)-\left(d_{j}^{(2)}\right)^{2}=0 .
$$

The solution to the quadratic equation above is

$$
\lambda=\lambda_{j}^{ \pm}=\frac{d_{j}^{(1)}+d_{j}^{(3)}}{2} \pm \sqrt{\left(\frac{d_{j}^{(1)}-d_{j}^{(3)}}{2}\right)^{2}+\left(d_{j}^{(2)}\right)^{2}}
$$

and the proof is complete.

Proof. of theorem 2,

Considering the conditions of theorem 2 we have by lemma 2 that the spectral bounds, $\alpha$ and $\beta$, satisfying

$$
0<\alpha=\min _{x \neq 0} \frac{x^{T} \underline{K} x}{x^{T} K x} \leq \lambda_{\min }\left(R^{-T} A R^{-1}\right) \leq \lambda_{\max }\left(R^{-T} A R^{-1}\right) \leq \max _{x \neq 0} \frac{x^{T} \bar{K} x}{x^{T} K x}=\beta,
$$

exist where $\underline{K}$ and $\bar{K}$ are defined in lemma 2 .

To find the exact values of $\alpha$ and $\beta$, we consider a generalized Rayleigh quotient

$$
Q(x)=\frac{x^{T} H x}{x^{T} K x}, \quad H=\left[\begin{array}{cc}
\delta_{1} K_{1} & M \\
M^{T} & \delta_{2} K_{2}
\end{array}\right],
$$

whose range is strictly positive. Thus, $Q(x)$ represents either $x^{T} \underline{K} x / x^{T} K x$ or $x^{T} \bar{K} x$. We apply the change of variables, $x=\left[\begin{array}{cc}R_{1}^{-T} & 0 \\ 0 & R_{2}^{-1}\end{array}\right] \xi$, and obtain the following equivalent representation

$$
Q(x)=\frac{\xi^{T} \widehat{H} \xi}{\xi^{T} \widehat{K} \xi}, \quad \widehat{H}=\left[\begin{array}{cc}
\delta_{1} I_{1} & \mathcal{M} \\
\mathcal{M}^{T} & \delta_{2} I_{2}
\end{array}\right], \quad \widehat{K}=\left[\begin{array}{cc}
I_{1} & \mathcal{M}_{r} \\
\mathcal{M}_{r}^{T} & I_{2}
\end{array}\right]
$$

where $\mathcal{M}=R_{1}^{-T} M R_{2}^{-1}$ and $\mathcal{M}_{r}=\mathcal{U}_{r} \Sigma_{r} \mathcal{V}_{r}^{T}$ is the $r$-rank weighted SVD approximation of $\mathcal{M}$.

Let $w_{i}$ denote the $i$-th column of the orthogonal matrix $\mathcal{W}$ eq. 2.12 as defined in lemma 1 . Then we have: 
1. $\widehat{K} w_{i}=\left(1+\sigma_{1}\right) w_{i}, i=1,2, \ldots, r$.

2. $\widehat{K} w_{n_{1}+i}=\left(1-\sigma_{1}\right) w_{n_{1}+i}, i=1,2, \ldots, r$.

3. $\widehat{K} w_{j}=w_{j}, j \neq 1, \ldots, r, n_{1}+1, \ldots, n_{1}+r$.

and similarly for $\widehat{H}$, it can be verified that:

1. $\widehat{H} w_{i}=\left(\delta_{1,2}^{\text {avg }}+\sigma_{1}\right) w_{i}+\delta_{1,2}^{\text {dif }} w_{n_{1}+i}, i=1,2, \ldots, \min \left\{n_{1}, n_{2}\right\}$.

2. $\widehat{H} w_{n_{1}+i}=\left(\delta_{1,2}^{\text {avg }}-\sigma_{1}\right) w_{n_{1}+i}+\delta_{1,2}^{\text {dif }} w_{i}, i=1,2, \ldots, \min \left\{n_{1}, n_{2}\right\}$.

3. $\widehat{H} w_{j}=w_{j}, j \neq 1, \ldots, \min \left\{n_{1}, n_{2}\right\}, n_{1}+1, \ldots, n_{1}+\min \left\{n_{1}, n_{2}\right\}$.

where $\delta_{1,2}^{\text {avg }}=\left(\delta_{1}+\delta_{2}\right) / 2$ and $\delta_{1,2}^{\text {dif }}=\left(\delta_{1}-\delta_{2}\right) / 2$. Clearly, both $\widehat{K}$ and $\widehat{H}$ are invariant over the subspaces $Z=\operatorname{span}\left\{w_{1}, \ldots, w_{r}, w_{n_{1}+1}, \ldots, w_{n_{1}+r}\right\}$ and its orthogonal complement, $Z^{\perp}$. Hence, by the properties of the generalized Rayleigh quotient we have:

$$
\max _{x \neq 0} Q(x)=\max \left\{\max _{\xi \in Z \backslash\{0\}} Q(x), \max _{\xi \in Z^{\perp} \backslash\{0\}} Q(x)\right\},
$$

and

$$
\min _{x \neq 0} Q(x)=\min \left\{\min _{\xi \in Z \backslash\{0\}} Q(x), \min _{\xi \in Z^{\perp} \backslash\{0\}} Q(x)\right\} .
$$

By our results so far, if $x=\xi \in Z^{\perp}$ then $Q(x)=\xi^{T} \widehat{H} \xi / \xi^{T} \xi$. Let us apply the change of variables of the form $\xi=C \zeta \in Z^{\perp}$, given explicitly by

$$
\xi=\zeta_{1} w_{r+1}+\ldots+\zeta_{n_{1}-r} w_{n_{1}}+\zeta_{n_{1}-r+1} w_{n_{1}+r+1}+\ldots+\zeta_{n_{1}+n_{2}-2 r} w_{n_{1}+n_{2}},
$$

where $\zeta_{i}$ is the $i$-th coordinate of $\zeta$ and as before $w_{i}$ denotes the $i$-th column in the orthogonal matrix $\mathcal{W}$ eq. 2.12). Then, for any $\xi \in Z^{\perp}$ we obtain

$$
Q(x)=\frac{\zeta^{T} \widehat{H}_{Z^{\perp} \zeta}}{\zeta^{T} \zeta}, \quad \widehat{H}_{Z^{\perp}}=\left[\begin{array}{cc}
\mathcal{D}_{Z \perp}^{(1)} & \mathcal{D}_{Z \perp}^{(2)} \\
\mathcal{D}_{Z^{\perp}}^{(2)^{T}} & \mathcal{D}_{Z^{\perp}}^{(3)}
\end{array}\right],
$$

where $\mathcal{D}_{Z^{\perp}}^{(2)}=\delta_{1,2}^{\text {dif }} I_{n_{1}, n_{2}}$ and

$$
\begin{aligned}
& \mathcal{D}_{Z^{\perp}}^{(1)}=\left\{\begin{array}{rrc}
\operatorname{diag}\left(\delta_{1,2}^{\mathrm{avg}}+\sigma_{r+1}, \ldots, \delta_{1,2}^{\text {avg }}+\sigma_{n_{1}}\right) & \text { if } & n_{1} \leq n_{2} \\
\operatorname{diag}\left(\delta_{1,2}^{\text {avg }}+\sigma_{r+1}, \ldots, \delta_{1,2}^{\text {avg }}+\sigma_{n_{1}}, \delta_{1}, \ldots, \delta_{1}\right) & \text { if } & n_{1}>n_{2}
\end{array} .\right. \\
& \mathcal{D}_{Z^{\perp}}^{(3)}=\left\{\begin{array}{rrr}
\operatorname{diag}\left(\delta_{1,2}^{\text {avg }}-\sigma_{r+1}, \ldots, \delta_{1,2}^{\text {avg }}-\sigma_{n_{2}}\right) & \text { if } & n_{2} \leq n_{1} \\
\operatorname{diag}\left(\delta_{1,2}^{\text {avg }}-\sigma_{r+1}, \ldots, \delta_{1,2}^{\text {avg }}-\sigma_{n_{2}}, \delta_{2}, \ldots, \delta_{2}\right) & \text { if } & n_{2}>n_{1}
\end{array} .\right.
\end{aligned}
$$

Now,by lemma 3 , we obtain that the spectrum of $\widehat{H}_{Z^{\perp}}$ contains the sets

$$
\begin{aligned}
& \left\{\delta_{1,2}^{\text {avg }}+\sqrt{\sigma_{r+1}^{2}+\left(\delta_{1,2}^{\text {dif }}\right)^{2}}, \ldots, \delta_{1,2}^{\text {avg }}+\sqrt{\sigma_{\min \left\{n_{1}, n_{2}\right\}}^{2}+\left(\delta_{1,2}^{\text {dif }}\right)^{2}},\right\}, \\
& \left\{\delta_{1,2}^{\text {avg }}-\sqrt{\sigma_{\min \left\{n_{1}, n_{2}\right\}}^{2}+\left(\delta_{1,2}^{\text {dif }}\right)^{2}}, \ldots, \delta_{1,2}^{\text {avg }}-\sqrt{\sigma_{r+1}^{2}+\left(\delta_{1,2}^{\text {dif }}\right)^{2}}\right\} .
\end{aligned}
$$

$\operatorname{RR} n^{\circ} 9200$ 
Hence, we conclude that

$$
\begin{gathered}
\min _{\xi \in Z^{\perp} \backslash\{0\}} Q(x)=\delta_{1,2}^{\text {avg }}-\sqrt{\sigma_{r+1}^{2}+\left(\delta_{1,2}^{\text {dif }}\right)^{2}}, \\
\max _{\xi \in Z^{\perp} \backslash\{0\}} Q(x)=\delta_{1,2}^{\text {avg }}+\sqrt{\sigma_{r+1}^{2}+\left(\delta_{1,2}^{\text {dif }}\right)^{2}} .
\end{gathered}
$$

For the case $\xi \in Z$ let us apply the change of variables of the form $\xi=C \psi \in Z$, given explicitly by

$$
\xi=\psi_{1} w_{1}+\ldots+\psi_{r} w_{r}+\psi_{r+1} w_{n_{1}+1}+\ldots+\psi_{2 r} w_{n_{1}+r}
$$

where $\psi_{i}$ is the $i$-th coordinate of $\psi$ and as before $w_{i}$ denotes the $i$-th column in the orthogonal matrix $\mathcal{W}$ eq. 2.12. Then, for any $\xi \in Z$ we obtain

$$
\begin{aligned}
& Q(x)=\frac{\psi^{T} \widehat{H}_{Z} \psi}{\psi^{T} \psi}, \quad \widehat{H}_{Z}=\left[\begin{array}{ll}
\mathcal{D}_{Z}^{(1)} & \mathcal{D}_{Z}^{(2)} \\
\mathcal{D}_{Z}^{(2)} & \mathcal{D}_{Z}^{(3)}
\end{array}\right] \\
& \mathcal{D}_{Z}^{(1)}=\operatorname{diag}\left(\frac{\delta_{1,2}^{\mathrm{avg}}+\sigma_{1}}{1+\sigma_{1}}, \ldots, \frac{\delta_{1,2}^{\mathrm{avg}}+\sigma_{r}}{1+\sigma_{r}}\right) \text {, } \\
& \mathcal{D}_{Z}^{(2)}=\operatorname{diag}\left(\frac{\delta_{1,2}^{\text {dif }}}{\sqrt{1-\sigma_{1}^{2}}}, \ldots, \frac{\delta_{1,2}^{\text {dif }}}{\sqrt{1-\sigma_{r}^{2}}}\right) \\
& \mathcal{D}_{Z}^{(3)}=\operatorname{diag}\left(\frac{\delta_{1,2}^{\mathrm{avg}}-\sigma_{1}}{1-\sigma_{1}}, \ldots, \frac{\delta_{1,2}^{\mathrm{avg}}-\sigma_{r}}{1-\sigma_{r}}\right) \text {. }
\end{aligned}
$$

Applying once more the outcome of lemma 3 we have that the spectrum of $\widehat{H}_{Z}$ is composed of the following values

$$
\frac{1}{2}\left(\frac{\delta_{1,2}^{\mathrm{avg}}+\sigma_{i}}{1+\sigma_{i}}+\frac{\delta_{1,2}^{\mathrm{avg}}-\sigma_{i}}{1-\sigma_{i}}\right) \pm \sqrt{\frac{1}{4}\left(\frac{\delta_{1,2}^{\mathrm{avg}}+\sigma_{i}}{1+\sigma_{i}}+\frac{\delta_{1,2}^{\mathrm{avg}}-\sigma_{i}}{1-\sigma_{i}}\right)^{2}+\frac{\left(\delta_{1,2}^{\mathrm{dif}}\right)^{2}}{1-\sigma_{i}^{2}}}
$$

where $i=1,2, \ldots, r$ and the proof is complete.

\section{Numerical Study}

This section contains the experimental part of this work. The main goal is to demonstrate the effect of different low-rank approximations eq. (3.2) for the off-diagonal blocks on the preconditioned system using HODLR. We perform a comparative study and consider the following low-rank techniques:

- R-HODLR: the off-diagonal low-rank factorizations are obtained in the standard or regular approach using truncated SVD.

- C-HODLR: the off-diagonal low-rank factorizations are obtained using truncated SVD with additional imposed constraints as described in [6].

- W-HODLR: the off-diagonal low-rank factorizations are obtained using the weighted HODLR preconditioning scheme for the multi-level case. The construction and application of the scheme follows the outlined procedure in section 3.3 . 
Employing SVD is done for convenience and uniformity of the comparison, and can be replaced, in practice, by other more efficient low-rank approximation techniques.

Section 4.1 describes a $2 D$ model problem whose matrix representation is numerically investigated in the section 4.2 in the context of hybrid domain decomposition methods. In section 4.2 we apply HODLR preconditioning schemes on a dense matrix which is obtained from the Schur complement of the model problem. The reduction of the problem to the Schur complement, essentially, ensures that HODLR techniques would be well suited to solve the reduced problem and, thus, allows us to perform accurate spectral analysis and demonstrate the better properties of the weighted low-rank approximation compared to the alternative low-rank techniques. More challenging severely ill-conditioned sparse systems are considered in section 4.3 . As in the previous subsection the weighted low-rank factorization scheme proves to be superior to other standard techniques.

\subsection{Poisson Problem with Robin Boundary Conditions}

Consider the 2D Poisson problem

$$
-u_{x x}-u_{y y}=f(x, y), \quad(x, y) \in(0,1) \times(0,1),
$$

with the following Robin boundary condition on the sides of the unit square, $\partial \Omega$,

$$
\left[\epsilon u+\partial_{\nu} u\right]_{\partial \Omega}=0
$$

where $\epsilon>0$ and $\partial_{\nu} u$ denotes the normal derivative. When $\epsilon \rightarrow 0^{+}$the problem becomes increasingly unstable since the limit case is the ill-posed Poisson problem with Neumann boundary conditions.

We discretize the problem by setting $(N+1)^{2}$ equally spaced gridpoints,

$$
x_{i}=\frac{i}{(2 N+2)}, \quad y_{j}=\frac{j}{(2 N+2)}, \quad i, j=1,2, \ldots, N+1
$$

and apply the five-point finite difference discretization rule. Thus, we end up with a symmetric linear system of $(N+1)^{2}$ equations in $(N+1)^{2}$ unknowns, $u_{i, j} \approx u\left(x_{i}, y_{j}\right)$. It can be shown, that the spectrum of the representing matrix is positive for all $\epsilon>0$. The system becomes singular when $\epsilon=0$. To avoid numerical instability we also scale the system and multiply it by $(N+1)^{2}$.

\subsection{Subdomain Preconditioning Simulation}

Let us consider a subdomain preconditioning problem which arises in hybrid domain decomposition methods for the solution of large scale problems. Hybrid methods employ direct and iterative techniques of solution and, thus, combine the advantages of both. Hybrid methods, essentially, employ three consecutive phases. First, the computational domain is split into smaller subdomains. In the second phase the Schur complement corresponding to the local interface of each local subsystem in each subdomain is computed by a direct solver. In the last phase the local Schur complements are assembled into the global Schur complement which is solved iteratively by an appropriate Krylov subspace technique. Some of the better known hybrid solver software packages are HIPS [14, MaPHyS [1], PDSLin [24, ShyLU [29], HPPDM [23, and PCBDDC 39 .

A robust approach for preconditioning the global Schur system is to employ a block preconditioner with overlap between the blocks, where each block is an approximate inverse of the locally 
assembled Schur complement of a corresponding subdomain. The block preconditioner is referred to as Algebraic Additive Schwarz (ADS). It is important to note that HODLR schemes are well suited for ADS preconditioning. This stems from the fact that the locally assembled Schur complement at the boundary nodes effectively represent a lower dimensional problem compared to the original problem. Thus, the effective rank of the off-diagonal blocks in the hierarchical partitioning is dramatically reduced. In particular, for a $2 \mathrm{D}$ problem the effective rank of each off-diagonal block, essentially, reduces to $\mathcal{O}(1)$. Hence, the inverse of a locally assembled Schur complement is expected to be well approximated by an HODLR approximation. For further information on ADS in the context of hybrid solvers, see [15].

Let us consider the 2D Poisson problem of section 4.1 as a sub-problem in a subdomain of a larger partitioned problem. Assuming a hybrid domain decomposition method is employed, we consider the Schur complement of the sub-problem which is defined on the subset of gridpoints eq. 4.2 that interact with the boundary of the computational domain,

$$
\left(x_{i}, y_{j}\right) \quad: \quad i=1 \text { or } j=N+1 \text { or } i=N+1 \text { or } j=1 .
$$

Obtaining the Schur system involves the factorization of the submatrix corresponding to the complement subset of gridpoints. We employ PASTIX [21], a sparse direct solver software, for computing the Schur complement, and a ScILAB 5.5.2 [32] implemented code to construct the HODLR preconditioners and to perform spectrum evaluations and PCG simulations. Numerical investigation shows that the condition number of the Schur system, $A$, satisfies $\operatorname{cond}(A) \sim 1 / \epsilon$, where $\epsilon>0$ is the control parameter of the Robin boundary condition eq. 4.1.

For constructing the HODLR preconditioning schemes we apply a balanced geometric partitioning on eq. (4.3) using successive bisections. We start by bisecting at the lower-left and upper-right corners. Next we bisect each subset at the lower-right and upper-left corners, respectively. The following bisections are applied to each subset of the partition, at the middle separating it into two equally sized subsets. We continue the process until reaching the predetermined bottom level of the hierarchy, $L$.

In all the simulations we have employed the following selection of the parameters:

- $N=10^{3}$ for the value of the grid parameter.

- $L=6$ as the lowest level of the hierarchy.

- $\epsilon=10^{-3}$ in the Robin b.c. eq. 4.1 for which $\operatorname{cond}(A) \approx 2.834 \cdot 10^{3}$.

For this selection of parameters, the dimension of the Schur complement matrix, $A$, is $4 \cdot 10^{3} \times 4 \cdot 10^{3}$ matrix, while the original sparse finite difference matrix is roughly a $10^{6} \times 10^{6}$ matrix. We denote the resulting Schur complement matrix by 'Subdomain1000'.

We approximate the low-rank off-diagonal blocks eq. (3.2) satisfying eq. (3.3) with a uniform truncation rule,

$$
\tau_{k}^{(\ell)}=\tau \quad \forall \ell=0,1, \ldots, L-1, k=1, \ldots, 2^{\ell},
$$

over the following range of values:

$$
\tau=0.4,0.2,0.1,0.05,0.025,0.005,0.002,0.001,0.0001 \text {. }
$$

To properly compare the preconditioners we examine their performance as a function of the global compression ratio, i.e., the percentage of the memory units kept by the HODLR approximation compared to the memory units required to store the dense Schur complement matrix.

The remainder of this subsection contains the numerical results of preconditioning the Schur complement dense problem, using R-HODLR, C-HODLR and W-HODLR schemes. Figure 5 
displays the spectrum clustering effect of the preconditioners. We display the spectrum of the preconditioned matrix for the three preconditioners when the global compression ratio is varied from about $7 \%$ up-to around $11 \%$. As expected, the higher the ratio the better the clustering of the eigenvalues around 1 . The results show that W-HODLR outperforms the other schemes achieving better spectrum clustering with fewer computational resources. Figure 6 shows the condition number of the preconditioned systems vs. the compression ratio. For small compression ratio, below $10 \%$, W-HODLR outperforms the other two variants achieving a better condition number with less memory resources. When more memory is used and the compression ratio increases above $12 \%$ all the variants yield almost the same condition number. Figure 7 portrays the number of iterations to convergence of the preconditioned conjugate gradient (PCG) method as a function of the compression ratio. The better conditioning observed in Figure 6 mostly translates in faster convergence, and W-HODLR performs the best compared to the two other variants for low value of the ratio (lower than 10\%). In the PCG simulations the right-hand-side was set to $b=1$, and the iterative approximation was stopped at the first occurrence of

$$
\left\|A x_{(i)}-b\right\|_{2} \leq 10^{-8}\|b\|_{2}
$$

where $i=1,2, \ldots$ is the iteration step index and $x_{(i)}$ denotes the approximate solution at step $i$. Figure 8 completes the results of fig. 7 with plots of the PCG convergence profiles. Clearly, the lower the value of the uniform tolerance $\tau$ eq. (4.4), the larger the rank and the faster the convergence rate. All the graphs exhibit steady monotonic decrease in logarithmic scale. This indicates that the preconditioned systems are not sensitive to the specific choice of the threshold in the stopping criterion eq. (4.5), and imply that regardless of the chosen threshold the features of fig. 7 would not dramatically change.

\subsection{Sparse Matrix Simulations}

In this subsection we numerically explore the PCG solution of the following sparse matrices:

- bcsstk16: $4,884 \times 4,884, \mathrm{SPD}$, spectral condition number $\approx 4.94 \cdot 10^{9}$.

- bcsstk15: $3,948 \times 3,948, \mathrm{SPD}$, spectral condition number $\approx 6.53 \cdot 10^{9}$.

- SparseRob500: 250,000 × 250,000, SPD, spectral condition number $\approx 1.08 \cdot 10^{6}$.

The first two are have been picked from the SuiteSparse matrix collection [10]. The third matrix is a realization of the sparse $2 \mathrm{D}$ Poisson problem of section 4.1 using $N=500$ for the value of the grid parameter.

For constructing the HODLR preconditioning schemes we interpret each matrix as a discrete graph and apply a balanced partitioning using successive bisections. We employ Scotch [9] for each bisection dividing a given vertex set into two distinct sets of approximately equal size whose cut is minimal, i.e., the number of edges running between the separated subsets is as small as possible. The process starts with the entire set of vertices, and then applied recursively on each separated subset until reaching the predetermined bottom level of the hierarchy, $L$.

Construction of the preconditioning schemes and the iterative solution of the preconditioned system has been implemented with a Fortran90 code. In all the simulations we have employed the following selections:

- $L=\left\lceil\log _{2}(n / 100)\right\rceil$ for an $n \times n$ matrix as the lowest level of the hierarchy, which forces the size of the smallest blocks in the partition under 100. 


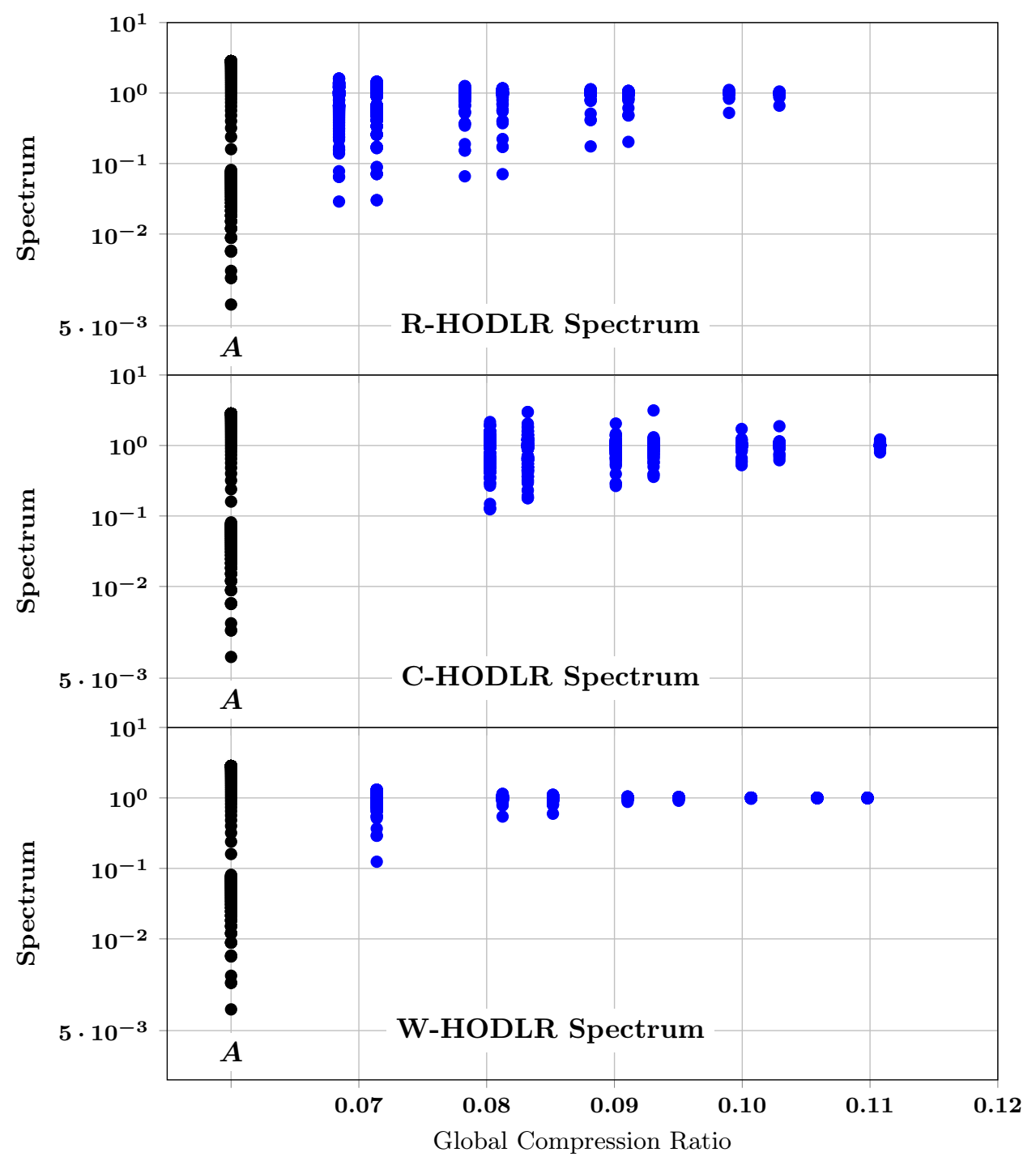

Figure 5: Spectrum Clustering: 'Subdomain1000'. Three plots showing the effect of the different low-rank factorization schemes on the spectrum of the preconditioned system. In each plot the spectra of the preconditioned system using each scheme are ordered from the left to the right starting from the spectrum of $A$ and then according to $\tau$ descending from the largest to the smallest value eq. 4.4. Note that C-HODLR memory consumption has an overhead cost due to the constraints it must satisfy.

- Constant off-diagonal block ranks over all levels of the hierarchy with the following $\mathcal{O}(1)$ values:

$$
r_{k}^{(\ell)}=0,5,10,15,20,25, \quad \ell=0,1, \ldots, L, \quad k=1, \ldots, 2^{\ell}
$$

Note that $r_{k}^{(\ell)} \equiv 0$ reduces the preconditioning scheme to block Jacobi (BJ), regardless of the specific low-rank factorization technique. 


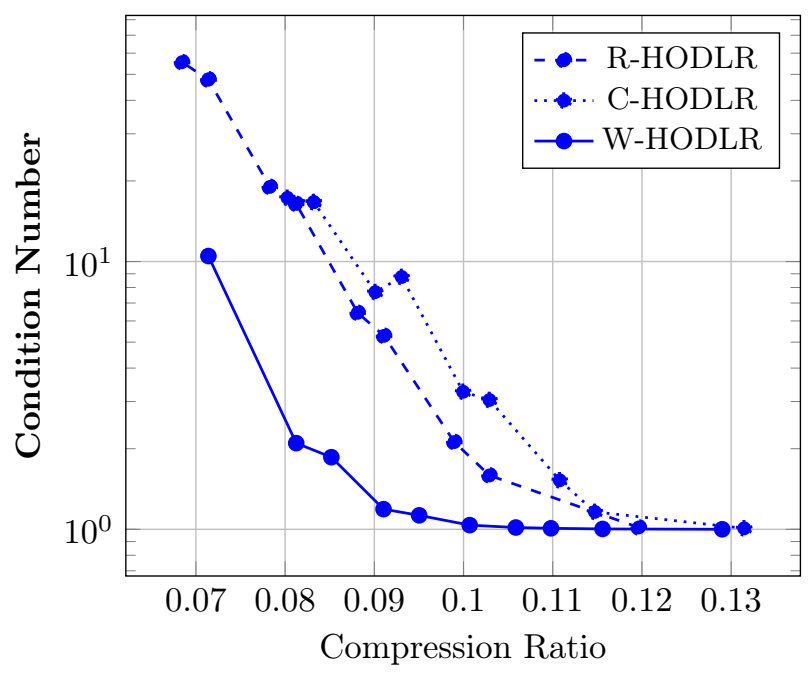

Figure 6: Condition Number vs. Compression: 'Subdomain1000'. Each plot shows the condition number achieved by an HODLR preconditioned system as a function of the compression ratio.

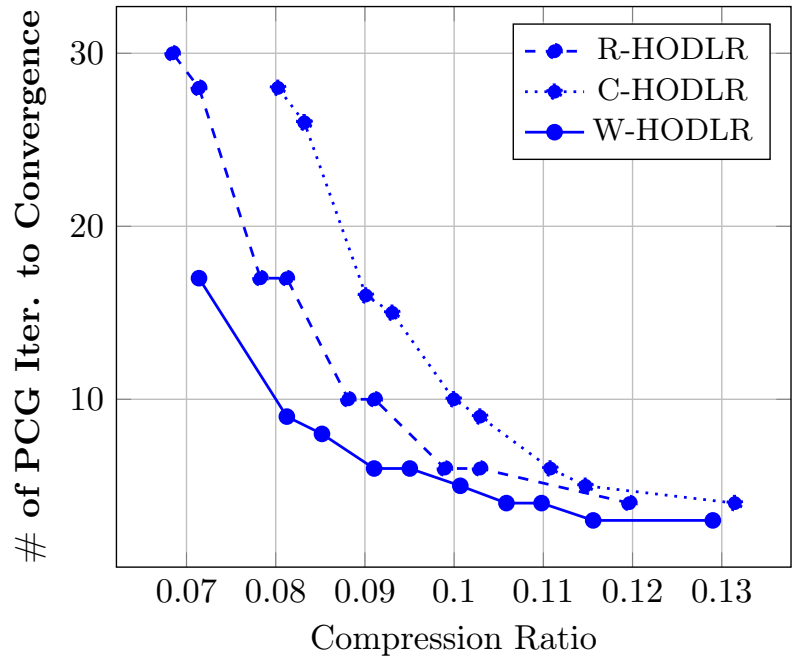

Figure 7: Iterations to Convergence vs. Compression: 'Subdomain1000'. Each plot shows the number of PCG iterations to convergence achieved by each HODLR preconditioned system as a function of the compression ratio.

The construction of the low-rank factorizations eq. (3.8) followed the path outlined in section 3.3. We have produced fast low-rank factorizations by first removing all the zero rows and columns of the sparse block $M_{k}^{(\ell)}$ eq. 3.4 , and then computing the low-rank factorization eq. 3.8 on the reduced block. For the sparse case this procedure is, typically, equivalent in terms of complexity to the randomized technique [26].

The remainder of this subsection contains the numerical results of PCG solution of the given 


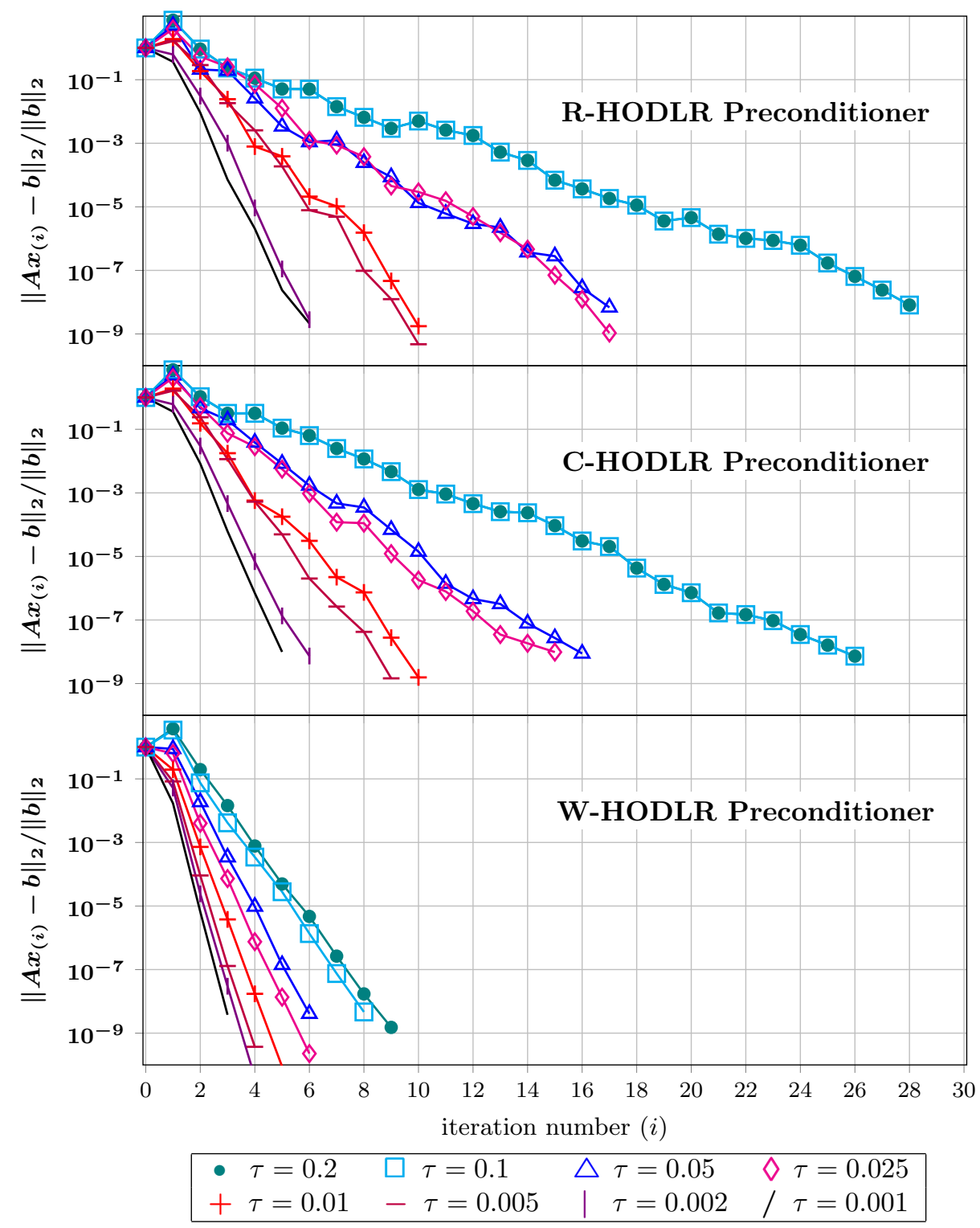

Figure 8: PCG Convergence History: 'Subdomain1000'. Three plots showing PCG convergence history profiles, i.e., the values $\left\|A x_{(i)}-b\right\|_{2} /\|b\|_{2}$ as a function of the iteration number $i$, for each preconditioning scheme. Each plot displays various profiles, where each profile corresponds to a different value eq. 4.4 of the uniform tolerance $\tau$ that is employed to set the off-diagonal bocks approximations by low-rank factorizations.

sparse systems, using R-HODLR, C-HODLR and W-HODLR preconditioning schemes. For bcsstk16 and bcsstk15 we have set the right-hand side to $b=1$, while for SparseRob500 we have chosen $b$ as the discretization of $\sin (x)$. We employ the same stopping criterion as before eq. 4.5. The results indicate that in all these example W-HODLR scheme outperforms the other techniques, and retain good properties even when low accuracy for the off-diagonal blocks 
approximations is employed.

Figure 9 contains plots of the PCG convergence history profiles for bcsstk16. All plots in this case indicate that increasing the constant rank eq. (4.6), improves the approximation quality, and achieves faster convergence rate. It is also evident that R-HODLR and C-HODLR achieve similar convergence with the same memory resources, while W-HODLR converges faster with the same memory resources. Figure 10 contains plots of the PCG convergence history profiles for bcsstk15. The results show that R-HODLR and C-HODLR fail to converge in 1000 PCG iterations. In fact, setting constant rank 0, i.e., Block Jacobi, performs better then using these schemes with a constant rank greater than zero. This occurs because the use of naive approximations for the off-diagonal blocks makes the problem even more ill-conditioned. The W-HODLR scheme, however, converges with excellent convergence rates, where the convergence rate improves when the constant rank eq. (4.6) is increased. Figure 10 contains plots of the PCG convergence history profiles for SparseRob500. The results show that R-HODLR converges much more slowly that W-HODLR, while C-HODLR fails to converge in 1000 PCG iterations. In this case it is evident that R-HODLR convergence does not depend monotonically on the constant rank value, and achieves better convergence than Block Jacobi (constant rank 0) only when the chosen constant rank is greater than 10. C-HODLR completely fails to produce a profile when the chosen constant rank is greater than 10, due to loss of the SPD property of the preconditioning scheme. Clearly, as in fig. 10 the results indicate that proper weighted approximations for the off-diagonal blocks are necessary to avoid disastrous outcome in terms of convergence.

\section{$5 \quad$ Summary and Future Work}

In this work we have addressed the problem of choosing low-rank factorizations in fast hierarchical algorithms for preconditioning SPD matrices.

We have presented a mathematical analysis for obtaining low-rank factorizations, that minimize the spectral condition number of the preconditioned system for the 1-level $(2 \times 2)$ case. The key idea was to properly reweight the blocks prior the low-rank factorization, which leads to a minimum spectral condition number.

The presented theory has been extended to HODLR preconditioning schemes, including analysis of the spectral properties and bounds that take into account the error propagation through the levels of the hierarchy.

The numerical experiments indicate, that employing proper reweighting for the off-diagonal blocks prior to low-rank compression, leads to a HODLR preconditioning scheme that requires far less computational resources for the same quality of performance of convergence than using the other low-rank compression techniques.

As noted in the introduction a major goal of this work is to provide an analysis of optimal choice of low-rank approximations for a simple case; i.e., HODLR, which could lead to an extended analysis for the strong hierarchical case. This point will be explored and pursued in a future study.

\section{Acknowledgments}

We would like to thank the anonymous referees for their valuable remarks, questions and comments that enabled us to substantially improve this paper. 


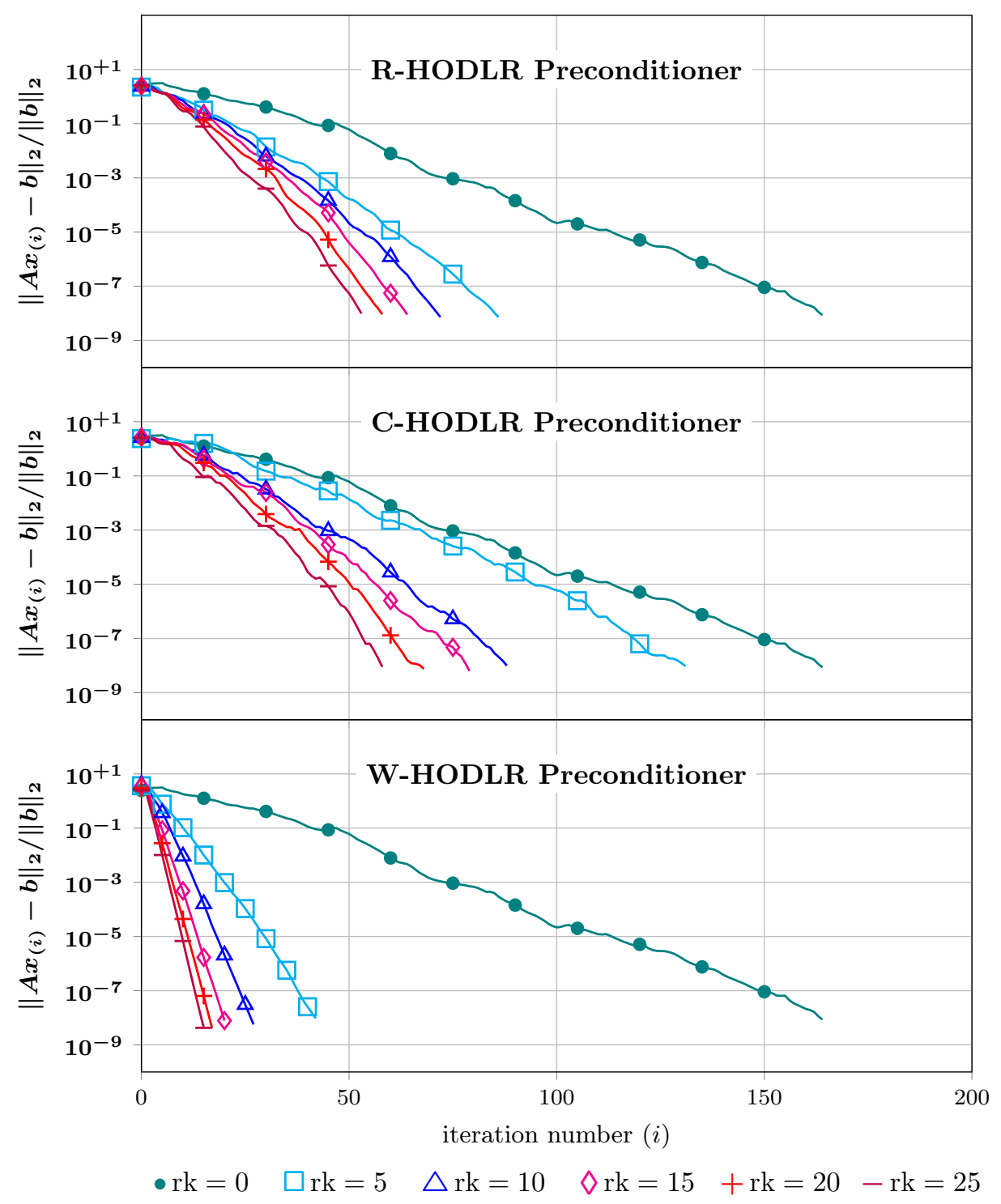

Figure 9: PCG Convergence History: 'bcsstk16'. Three plots showing PCG convergence history profiles, i.e., the values $\left\|A x_{(i)}-b\right\|_{2} /\|b\|_{2}$ as a function of the iteration number $i$, for each preconditioning scheme. Each plot displays various profiles, where each profile corresponds to a different constant rank value eq. 4.6. of the approximations for the off-diagonals blocks by low-rank factorizations. 


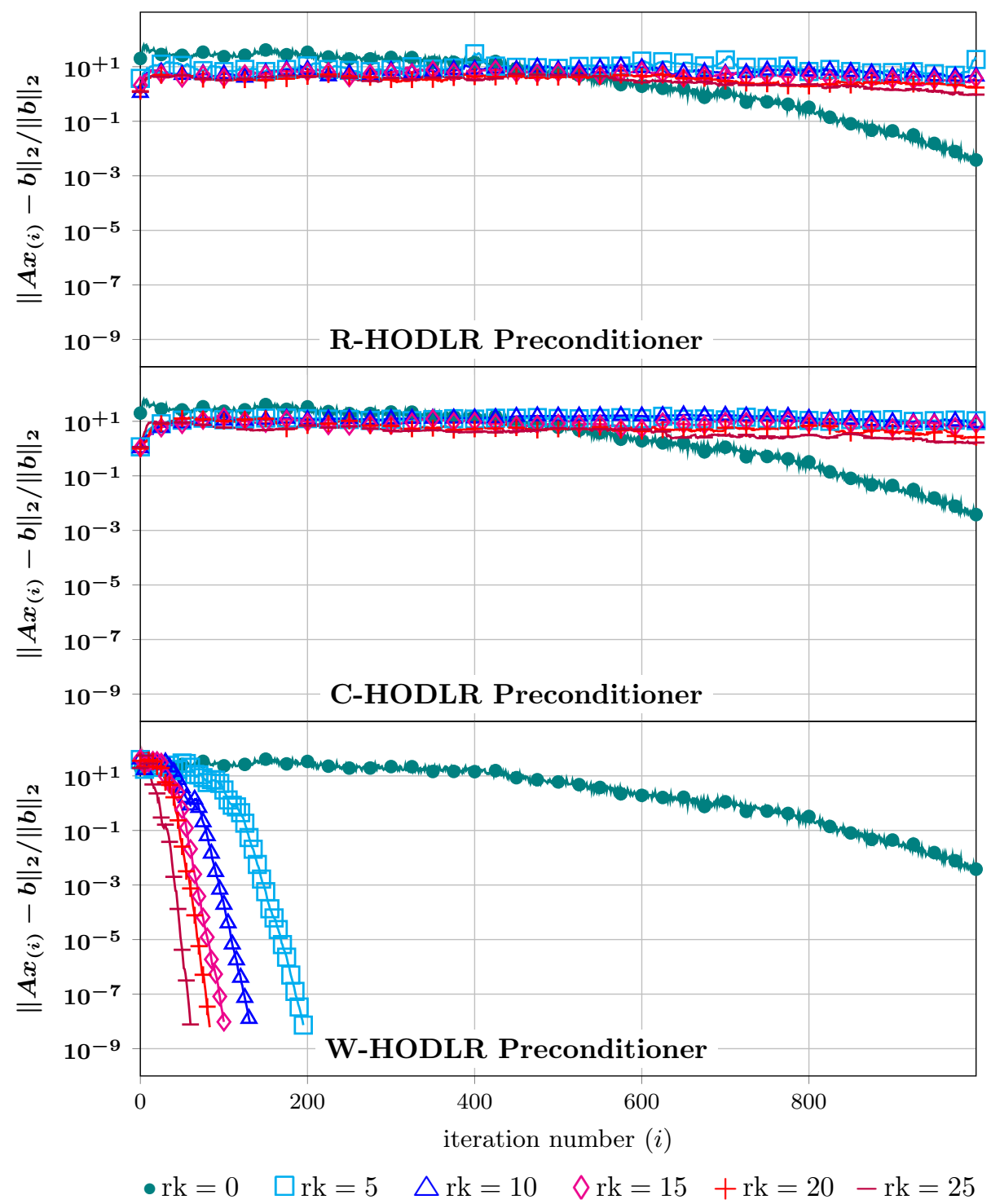

Figure 10: PCG Convergence History: 'bcsstk15'. Three plots showing PCG convergence history profiles, i.e., the values $\left\|A x_{(i)}-b\right\|_{2} /\|b\|_{2}$ as a function of the iteration number $i$, for each preconditioning scheme. Each plot displays various profiles, where each profile corresponds to a different constant rank value eq. 4.6 of the approximations for the off-diagonals blocks by low-rank factorizations. 


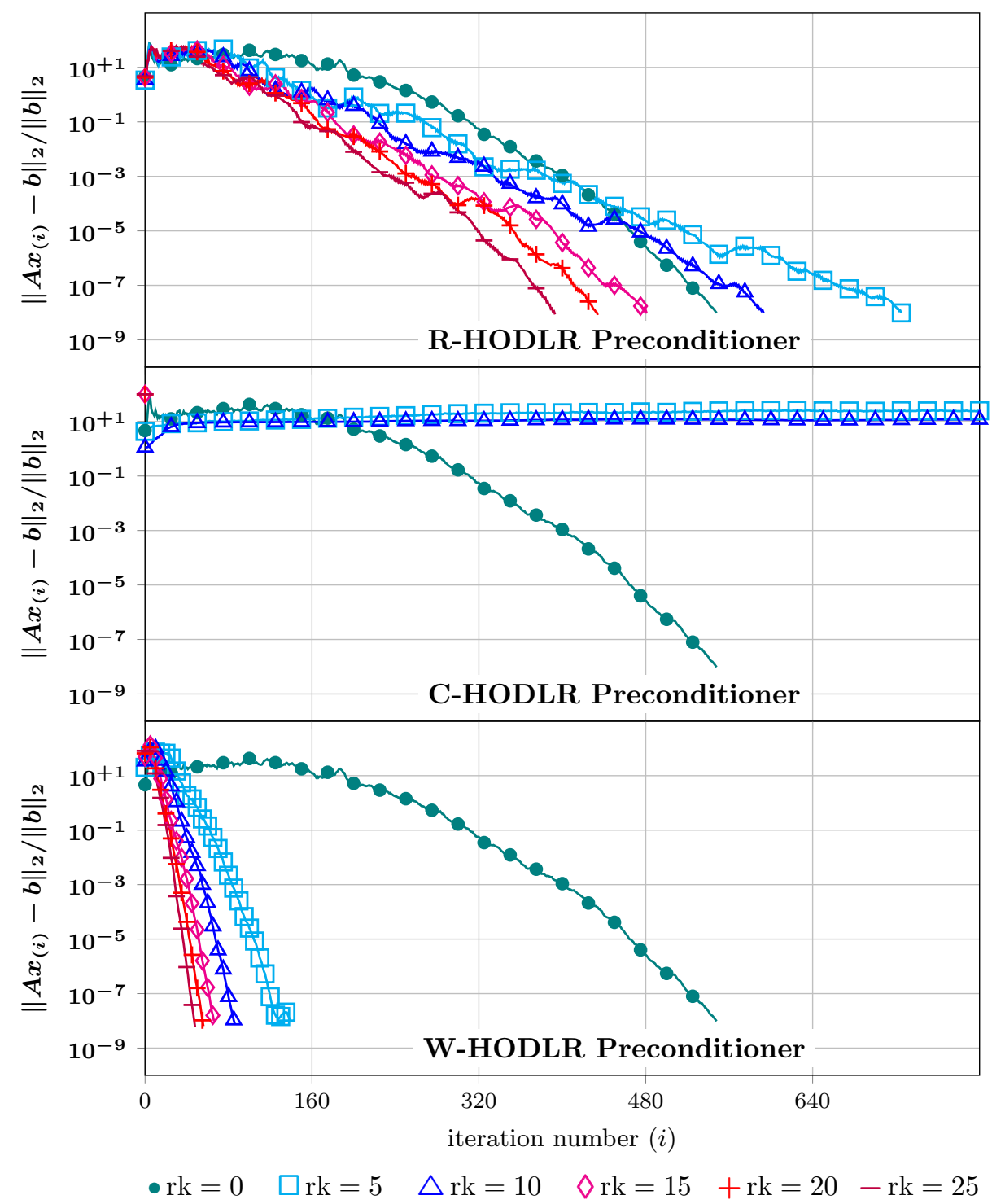

Figure 11: PCG Convergence History: 'SparseRob500'. Three plots showing PCG convergence history profiles, i.e., the values $\left\|A x_{(i)}-b\right\|_{2} /\|b\|_{2}$ as a function of the iteration number $i$, for each preconditioning scheme. Each plot displays various profiles, where each profile corresponds to a different constant rank value eq. 4.6 of the approximations for the off-diagonals blocks by low-rank factorizations. 


\section{References}

[1] E. Agullo, L. Giraud, A. Guermouche, and J. Roman, Parallel hierarchical hybrid linear solvers for emerging computing platforms, Comptes Rendus Mécanique, 339 (2011), pp. 96 - 103. High Performance Computing.

[2] A. Aminfar, S. Ambikasaran, And E. Darve, A fast block low-rank dense solver with applications to finite-element matrices, Journal of Computational Physics, 304 (2016), pp. 170 $-188$.

[3] O. Axelsson And P. S. Vassilevski, Algebraic multilevel preconditioning methods. I, Numerische Mathematik, 56 (1989), pp. 157-177.

[4] O. Axelsson and P. S. Vassilevski, Algebraic Multilevel Preconditioning Methods, II, SIAM Journal on Numerical Analysis, 27 (1990), pp. 1569-1590.

[5] M. Bebendorf, Hierarchical Matrices: A Means to Efficiently Solve Elliptic Boundary Value Problems, Springer Berlin Heidelberg, 2008.

[6] M. Bebendorf, M. Bollhöfer, And M. Bratsch, Hierarchical matrix approximation with blockwise constraints, BIT Numerical Mathematics, 53 (2013), pp. 311-339.

[7] M. Bebendorf, M. Bollhöfer, And M. Bratsch, On the spectral equivalence of hierarchical matrix preconditioners for elliptic problems, Mathematics of Computation, 85 (2016), pp. 2839-2861.

[8] S. Chandrasekaran, M. Gu, and T. Pals, A Fast ULV Decomposition Solver for Hierarchically Semiseparable Representations, SIAM Journal on Matrix Analysis and Applications, 28 (2006), pp. 603-622.

[9] C. Chevalier and F. Pellegrini, PT-Scotch: A tool for efficient parallel graph ordering, Parallel Computing, 34 (2008), pp. 318 - 331. Parallel Matrix Algorithms and Applications.

[10] T. A. Davis And Y. Hu, The University of Florida Sparse Matrix Collection, ACM Trans. Math. Softw., 38 (2011), pp. 1:1-1:25.

[11] C. Eckart And G. Young, The approximation of one matrix by another of lower rank, Psychometrika, 1 (1936), pp. 211-218.

[12] L. Elsner, A note on optimal block-scaling of matrices, Numerische Mathematik, 44 (1984), pp. $127-128$.

[13] A. Frieze, R. Kannan, And S. Vempala, Fast Monte-carlo Algorithms for Finding Lowrank Approximations, J. ACM, 51 (2004), pp. 1025-1041.

[14] J. Gaidamour And P. HÉnOn, A parallel direct/iterative solver based on a schur complement approach, in 11th IEEE International Conference on Computational Science and Engineering, 2008, CSE'08, IEEE Computer Society, 2008, pp. 98-105.

[15] L. Giraud, A. Haidar, And L. Watson, Parallel scalability study of hybrid preconditioners in three dimensions, Parallel Computing, 34 (2008), pp. 363 - 379. Parallel Matrix Algorithms and Applications.

[16] G. H. Golub And C. F. V. Loan, Matrix computations, Forth Edition, Johns Hopkins University Press, 2013.

$\operatorname{RR} \mathrm{n}^{\circ} 9200$ 
[17] L. Grasedyck and W. Hackbusch, Construction and Arithmetics of H-Matrices, Springer Journal of Computing, 70 (2003), pp. 295-334.

[18] M. Gu And S. C. Eisenstat, Efficient Algorithms for Computing a Strong Rank-Revealing QR Factorization, SIAM Journal on Scientific Computing, 17 (1996), pp. 848-869.

[19] W. Hackbusch, A Sparse Matrix Arithmetic Based on $\mathcal{H}$-Matrices. Part I: Introduction to $\mathcal{H}$-Matrices, Computing, 62 (1999), pp. 89-108.

[20] W. Hackbusch and B. N. KhoromskiJ, A Sparse $\mathcal{H}$-Matrix Arithmetic. Part II: Application to Multi-Dimensional Problems, Computing, 64 (2000), pp. 21-47.

[21] P. Hénon, P. Ramet, And J. Roman, PaStiX: A High-Performance Parallel Direct Solver for Sparse Symmetric Definite Systems, Parallel Computing, 28 (2002), pp. 301-321.

[22] M. R. Hestenes And E. Stiefel, Methods of conjugate gradients for solving linear systems, Journal of Research of the National Bureau of Standards, 49 (1952).

[23] P. Jolivet, F. Hecht, F. Nataf, And C. Prud'homme, Scalable Domain Decomposition Preconditioners for Heterogeneous Elliptic Problems, in Proceedings of the International Conference on High Performance Computing, Networking, Storage and Analysis, SC '13, New York, NY, USA, 2013, ACM, pp. 80:1-80:11.

[24] X. S. Li, M. Shao, I. Yamazaki, and E. G. NG, Factorization-based sparse solvers and preconditioners, Journal of Physics: Conference Series, 180 (2009), p. 012015.

[25] E. Liberty, F. Woolfe, P.-G. Martinsson, V. Rokhlin, and M. Tygert, Randomized algorithms for the low-rank approximation of matrices, Proceedings of the National Academy of Sciences, 104 (2007), pp. 20167-20172.

[26] P. G. Martinsson, A Fast Randomized Algorithm for Computing a Hierarchically Semiseparable Representation of a Matrix, SIAM Journal on Matrix Analysis and Applications, 32 (2011), pp. 1251-1274.

[27] L. Miranian and M. Gu, Strong rank revealing LU factorizations, Linear Algebra and its Applications, 367 (2003), pp. $1-16$.

[28] B. Parlett, The Symmetric Eigenvalue Problem, Society for Industrial and Applied Mathematics, 1998.

[29] S. Rajamanickam, E. G. Boman, and M. A. Heroux, ShyLU: A Hybrid-Hybrid Solver for Multicore Platforms, in Proceedings of the 2012 IEEE 26th International Parallel and Distributed Processing Symposium, IPDPS '12, Washington, DC, USA, 2012, IEEE Computer Society, pp. 631-643.

[30] S. RJasanow, Adaptive cross approximation of dense matrices, in Int. Association Boundary Element Methods Conf., IABEM, 2002, pp. 28-30.

[31] Y. SaAd, Iterative Methods for Sparse Linear Systems, Second Edition, Siam, 2003.

[32] Scilab Enterprises, Scilab: Free and Open Source software for numerical computation, Scilab Enterprises, Orsay, France, 2012.

[33] A. J. Wathen, Preconditioning, Acta Numerica, 24 (2015), pp. 329-376. 
[34] F. Woolfe, E. Liberty, V. Rokhlin, And M. Tygert, A fast randomized algorithm for the approximation of matrices, Applied and Computational Harmonic Analysis, 25 (2008), pp. $335-366$.

[35] J. Xia, S. Chandrasekaran, M. GU, And X. S. Li, Fast algorithms for hierarchically semiseparable matrices, Numerical Linear Algebra with Applications, 17 (2010), pp. 953976.

[36] J. Xia And M. Gu, Robust Approximate Cholesky Factorization of Rank-Structured Symmetric Positive Definite Matrices, SIAM Journal on Matrix Analysis and Applications, 31 (2010), pp. 2899-2920.

[37] J. XIA AND Z. XIN, Effective and Robust Preconditioning of General SPD Matrices via Structured Incomplete Factorization, SIAM Journal on Matrix Analysis and Applications, 38 (2017), pp. 1298-1322.

[38] K. Yang, H. Pouransari, and E. Darve, Sparse Hierarchical Solvers with Guaranteed Convergence, ArXiv e-prints, (2016), arXiv:1611.03189.

[39] S. Zampini, PCBDDC: A Class of Robust Dual-Primal Methods in PETSc, SIAM Journal on Scientific Computing, 38 (2016), pp. S282-S306. 


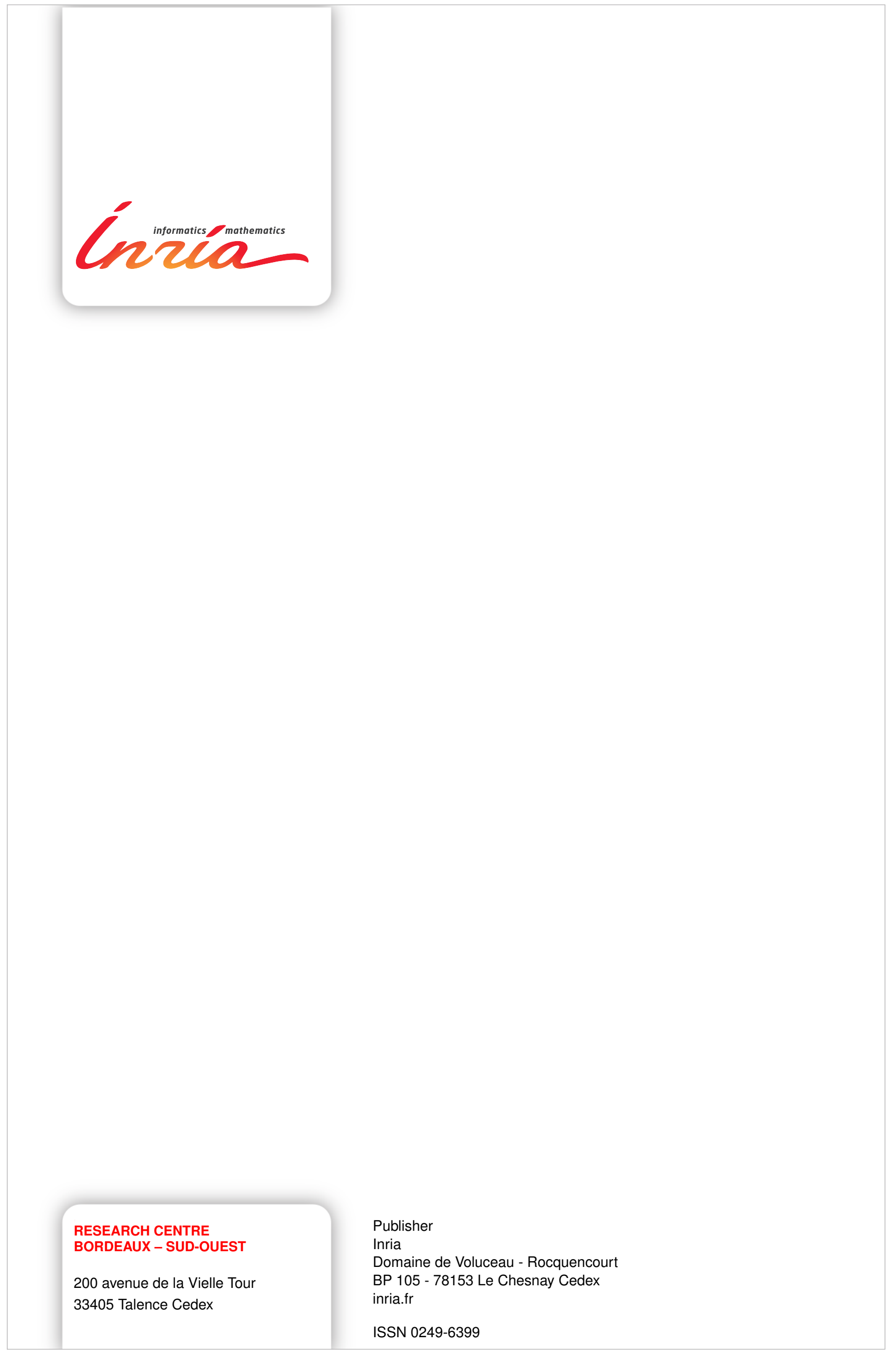

